\title{
A Numerical Study of Lean Propane-Air Flame Acceleration at the Early Stages of Burning in Cold and Hot Isothermal Walled Small-Size Tubes
}

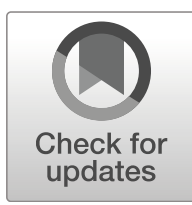

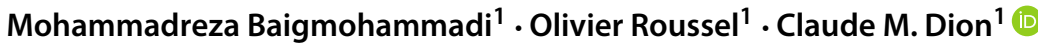

Received: 22 June 2018 / Accepted: 30 July 2019 / Published online: 3 September 2019

(C) The Author(s) 2019

\begin{abstract}
In this study, the problem of lean propane-air premixed flame acceleration from closed to open end during the early stages of burning in small-size tubes with isothermal walls was considered. In particular, the effects of tube radius, slip/non-slip wall conditions, and the wall temperature on the flame propagation and shape were investigated numerically. Five stages of flame propagation are identified: 1) spherical expansion of the flame front; 2) finger shape expansion of the flame front before touching the wall; 3) flame propagation in the tube subjected to flame-wall interactions; 4) transformation of the flame shape into tulip form; 5) conversion of the tulip shape flame to finger. Our results show that the tube radius, wall condition and its temperature significantly affect flame propagation regimes even in the first instance of the flame propagation in the tubes. We find that increasing tube radius has, overall, the effect of increasing the flame propagation speed in isothermal tubes. Also, depending on tube radius and wall condition, the wall temperature can increase or decrease the flame propagation speed in the isothermal tubes. Furthermore, the results demonstrate that imposing either slip or non-slip condition on the tube's walls impressively affects flame acceleration and its configuration in the early stages. We observe that, unlike flame propagation forms in the tubes with slip walls, the early exponential flame propagation phase in the tubes was generally followed by a linear flame propagation phase in the tubes with a non-slip wall condition. We obtain that flame propagation in tubes with slip wall conditions are more sensitive to variations in tube radius and wall temperature compared to non-slip conditions. We also see that, contrary to the exponential flame propagation phase, increasing the non-slip wall temperature reduces the flame propagation speed in the linear part of the flame propagation, while such an increase in temperature leads to oscillations in the flame propagation speed in the tubes with slip walls.
\end{abstract}

Keywords Flame acceleration · Narrow tubes · Propane-air · Isothermal walls · Cold walls $\cdot$ Hot walls

Claude M. Dion

claude.dion@umu.se

1 Department of Physics, Umeå University, SE-901 87 Umeå, Sweden 


\section{Nomenclature}

\section{English}

$A_{\mathrm{S}} \quad$ Sutherland coefficient

$C_{p} \quad$ heat capacity at constant pressure $\left(\mathrm{J} \mathrm{kg}^{-1} \mathrm{~K}^{-1}\right)$

$D$ mass diffusivity $\left(\mathrm{m}^{2} \mathrm{~s}^{-1}\right)$

$E_{\mathrm{a}} \quad$ activation energy $\left(\mathrm{J} \mathrm{mol}^{-1}\right)$

$f \quad$ Force applied on the fluid

$H^{\circ} \quad$ state-standard molar enthalpy $\left(\mathrm{J} \mathrm{mol}^{-1}\right)$

$h_{\mathrm{s}} \quad$ sensible enthalpy $\left(\mathrm{J} \mathrm{mol}^{-1}\right)$

$K$ thermal conductivity $\left(\mathrm{W} \mathrm{m}^{-1} \mathrm{~K}^{-1}\right)$

$k \quad$ rate constant

$L \quad$ tube length (m)

$L_{\mathrm{f}} \quad$ flame thickness $(\mathrm{m})$

Le Lewis number $(=\alpha / D)$

$M \quad$ molecular weight $\left(\mathrm{kg} \mathrm{mol}^{-1}\right)$

$\bar{M} \quad$ average molecular weight of mixture $\left(\mathrm{kg} \mathrm{mol}^{-1}\right)$

$p \quad$ pressure $(\mathrm{Pa})$

$\dot{Q} \quad$ heat source term (W)

$R \quad$ tube radius (m)

$R_{\mathrm{u}} \quad$ universal gas constant $\left(\mathrm{J} \mathrm{mol}^{-1} \mathrm{~K}^{-1}\right)$

$\mathrm{Re} \quad$ Reynolds number

$S^{\circ} \quad$ state-standard molar entropy $\left(\mathrm{J} \mathrm{K}^{-1} \mathrm{~mol}^{-1}\right)$

$T \quad$ temperature (K)

$T_{\mathrm{a}} \quad$ activation temperature $(\mathrm{K})$

$T_{\mathrm{S}} \quad$ Sutherland temperature $(\mathrm{K})$

$U_{\mathrm{f}} \quad$ laminar flame speed $\left(\mathrm{m} \mathrm{s}^{-1}\right)$

$u \quad$ velocity $\left(\mathrm{m} \mathrm{s}^{-1}\right)$

$V^{c} \quad$ correction velocity $\left(\mathrm{m} \mathrm{s}^{-1}\right)$

$W \quad$ Thermophoretic diffusion velocity $\left(\mathrm{m} \mathrm{s}^{-1}\right)$

$X \quad$ mole fraction

$Y \quad$ mass fraction

$Z_{\text {tip }} \quad$ position of the flame along the tube (m)

Greek

$\alpha \quad$ thermal diffusivity $\left(\mathrm{m}^{2} \mathrm{~s}^{-1}\right)$

$\delta_{i j} \quad$ Kronecker delta

$\delta_{\mathrm{t}} \quad$ flame thickness (m)

$\varepsilon \quad$ Lennard-Jones energy (J)

$\Phi$ equivalence ratio

$\mu \quad$ dynamic viscosity $\left(\mathrm{N} \mathrm{s} \mathrm{m}^{-2}\right)$

$\rho \quad$ density $\left(\mathrm{kg} \mathrm{m}^{-3}\right)$

$\sigma \quad$ collision diameter [Lennard-Jones characteristic length] ( $\AA$ )

$\tau \quad$ dimensionless time

$\Theta \quad$ thermal expansion coefficient

$\xi_{\text {tip }} \quad$ flame tip position (dimensionless)

$\Omega_{D}$ analogous reduced collision integral

$\dot{\omega} \quad$ Species mass generation rate per unit volume $\left(\mathrm{kg} \mathrm{m}^{-3} \mathrm{~s}^{-1}\right)$ 


\begin{tabular}{ll}
\multicolumn{2}{l}{ Subscripts } \\
0 & initial values \\
$\mathrm{b}$ & burnt \\
$i, j, k$ & directions or species \\
mix & mixture \\
$\mathrm{S}$ & Sutherland coefficients \\
$\mathrm{u}$ & unburnt \\
$\mathrm{w}$ & wall
\end{tabular}

\section{Introduction}

Predicting flame shape and acceleration in confined spaces and chambers is vitally important for safety issues and designing meso- and micro-scale combustors. In terms of safety issues, it stands behind many disasters like explosions in pressure vessels, power plants, and mining accidents producing heavy damage and costing hundreds of lives almost annually [1]. Also, designing reliable and efficient meso- and micro-scale combustors/reactors highly depends on the accurate prediction of flame shape and acceleration in small size combustors. In fact, it could help designers to better understand and control hydrodynamic and thermodiffusive instabilities in small-scale combustors and consequently increase their combustion efficiency. In this regard, the most typical geometry in considering safety issues and energyproduction devices corresponds to relatively long channels, with the flame propagating from a closed channel end to an open one.

The first qualitative explanation of flame acceleration in tubes with smooth walls was suggested by Shelkin in the 1940's (see, e.g., [1-4]). Shelkin related the flame acceleration to non-slip conditions at the tube walls and to flow turbulence. Burning gas expands within the flame front and generates a flow in the fuel mixture. According to the Shelkin mechanism, non-slip channel walls make the flow non-uniform, which bends the flame, increases the burning rate, and leads to a stronger flow. The positive feedback between the flame and the flow results in flame acceleration.

It was shown in [5] that the early stage of the flame propagation in small size tubes is divided into three distinct parts: (1) propagation at a constant velocity; (2) deceleration; (3) exponential acceleration. It was found that the early stages of flame propagation have a determining role in flame propagation scenarios in small-sized tubes. In research reported in [6], it was shown that flame acceleration in an adiabatic tube with slip wall conditions is strongly dependent on the initial ignition geometry. This finding was also confirmed in $[7,8]$, which showed how different ignition methods could affect the flame shape and its propagation regime in such tubes/channels. In other research [9], it was demonstrated that flame acceleration in adiabatic channels can be intensified by increasing the thermal expansion coefficient $\Theta$ and decreasing the tube diameter (or Reynolds number). Also, it was shown in [10] that the minimum width of the preheated zone that may lead to supersonic flame front speed was about 17-25 times the laminar flame thickness.

In a series of theoretical and numerical studies reported in [11-13], it was demonstrated that, independent of the channel height, the flame accelerates at a nearly constant rate during the early stages of propagation. They confirmed that premixed flames in long, finite channels could self-accelerate, so that their propagation velocities may reach values which are ten to twenty times larger than the laminar flame speed [11]. Moreover, it was shown that the imposed boundary conditions at the end of a channel have a significant effect on 
the flame propagation modes [13]. This research also indicated that the boundary condition at the ignition place can affect the flame propagation regime in adiabatic channels [12].

Moreover, it was reported in [14] that the thermal boundary conditions and the channel height play critical roles in the propagation scenarios of premixed acetylene-air flames in small-sized channels, such that increasing the channel height may increase the possibility of formation of tulip-shaped flame fronts in such channels. Also, it was shown in [15] that the presence of a perturbation on the flame surface plays a crucial role in the flame acceleration in the tubes. Other experimental research [16] found that, depending on mixture composition and the length of tube, pressure oscillation may lead to an oscillatory effect on the flame propagation trajectory in the tube. In addition, it was investigated in $[17,18]$ that, depending on the tube diameter and the deviation in temperature, the effect of the temperature profile on the flame propagation speed and flame shape can be significant. Also, imposing non-slip conditions on walls could double the flame propagation velocity with respect to slip conditions under elevated temperature cases.

As mentioned above, to obtain a fundamental understanding and attain the technological control of flame evolution in small size tubes, a reliable quantitative model of flame acceleration is still greatly needed. However, until now, most of theoretical and numerical work on flame evolution in small-sized channels/tubes have been performed within the simplified model of channels/tubes with either negligible heat loss to the walls or almost cold isothermal wall conditions. While this has allowed for progress in understanding the basic mechanisms and properties of flame evolution, the actual process involves inevitable losses. Also, according to the literature, it can be found that although various boundary conditions (such as different velocity profiles, slip/non-slip conditions on the inner walls, and constant wall temperature) have been imposed on flow fields in the tubes, the effects of elevated wall temperatures or the early stages of flame propagation from a small size kernel to a propagating flame front in isothermal wall tubes have not been studied. In fact, in the various studies cited above, the cumulative effects of the studied parameters on the early stages of fuel-lean flame propagation in small-sized, one-end closed isothermal tubes with cold/hot walls and slip/non-slip wall conditions have not been investigated simultaneously in a research paper.

In this regard, we present here a detailed numerical investigation of the effects of isothermal elevated wall temperature $(\gg 300 \mathrm{~K}$ ), tube radius, and wall condition (slip/non-slip) on the early stages of the flame evolution process in one-end closed tubes. In particular, we consider the simultaneous effects of various boundary conditions on flame evolution pattern in small-sized, one-closed end tubes. Focusing on micro-combustion schemes, the purpose of the present work is to develop a numerical understanding of the early stages of flame acceleration into a real design tool for predicting the key flame acceleration stages. By studying the early stages of flame evolution in small size one-closed end tubes, the understanding gained here may be useful for optimization of DDT (Deflagration-to-Detonation Transition)-based devices of energy production, such as small-sized pulse-detonation engines and micro-combustion devices, and improving energy safety.

In the following, we first briefly present the theory behind the problem at hand. Section 3 then details the numerical approach used, which is validated in Section 4. The results and presented and analyzed in Section 5, and we end the paper with a summary and conclusions.

\section{Theory}

In this section, we present an overview of the results of the theory of the early stages of burning of a premixed hydrocarbon fuel-air flame in a one-end closed adiabatic tube, as 
developed by Bychkov et al. [6]. Only the resulting equations are presented here, and we refer the reader to [6] for a detailed description of the methodologies and assumptions used. For the expansion factor

$$
\Theta=\frac{\rho_{\mathrm{u}}}{\rho_{\mathrm{b}}}=\frac{T_{\mathrm{b}}}{T_{\mathrm{u}}},
$$

the dimensionless time

$$
\tau \equiv \frac{U_{\mathrm{f}} t}{R}
$$

and

$$
\alpha \equiv \sqrt{\Theta(\Theta-1)},
$$

one gets the flame tip position

$$
\xi_{\text {tip }}=\frac{\Theta}{2 \alpha} \sinh (2 \alpha \tau),
$$

the time when the flame front propagates spherically

$$
\tau_{\mathrm{sph}} \approx \frac{1}{2 \alpha}
$$

and the time when the flame front touches the walls

$$
\tau_{\text {wall }}=\frac{1}{2 \alpha} \ln \left(\frac{\Theta+\alpha}{\Theta-\alpha}\right) .
$$

As mentioned in [6], these solutions describe the flame front behavior during the $\tau_{\text {sph }}<\tau<\tau_{\text {wall }}$ time interval. The above results, accompanied by the available experimental data [19], will be applied in the following for validating the numerical results obtained in the present study.

\section{Numerical Modeling}

A schematic view of the applied physical domain in the current simulations is depicted in Fig. 1a. As can be seen, the length of the applied closed-end tube, $L$, is 60 times the tube radius $R$. For the sake of considering the probable effect of the tube length on the flame propagation in the tube, another tube with length of $100 R$ is also modeled and its results have been compared with the results of a tube $60 R$ in length. A detailed discussion in this regard is presented in Section 4. Also, the tube radius is expressed relative to the preheat zone thickness of the unstretched laminar flame of the applied fuel-lean propane-air mixture under adiabatic conditions,

$$
L_{\mathrm{f}}=\frac{K_{\text {mix }}}{\rho_{\text {mix }} C_{p, \text { mix }} U_{\mathrm{f}}},
$$

with tube radii $R=10 L_{\mathrm{f}}, 20 L_{\mathrm{f}}$, and $30 L_{\mathrm{f}}$.

As implicitly shown in Fig. $1 \mathrm{~b}$ and presented in Table 1, the tube was filled with a non-stoichiometric, premixed propane-air mixture with equivalence ratio $\Phi=0.7$. The value of the unstretched laminar adiabatic flame speed $U_{\mathrm{f}}$ in these conditions is taken as $0.217 \mathrm{~m} / \mathrm{s}$ [20]. According Eq. 7 and the applied reactive mixture [6, 19, 20], the thickness of the flame preheat zone was calculated as $L_{\mathrm{f}}=8.34 \times 10^{-5} \mathrm{~m}$. Due to the axisymmetric geometry of the tube, and for the sake of reducing the computation cost, only one half of the tube was modeled. Thus, it is seen in Fig. $1 \mathrm{~b}$ that the tube is modeled as a wedge with the wedge angle of only $2^{\circ}$, eliminating $3 \mathrm{D}$ flow field motion effects on the results. These wedge patches are empty surfaces which simulate the axisymmetric condition in the 


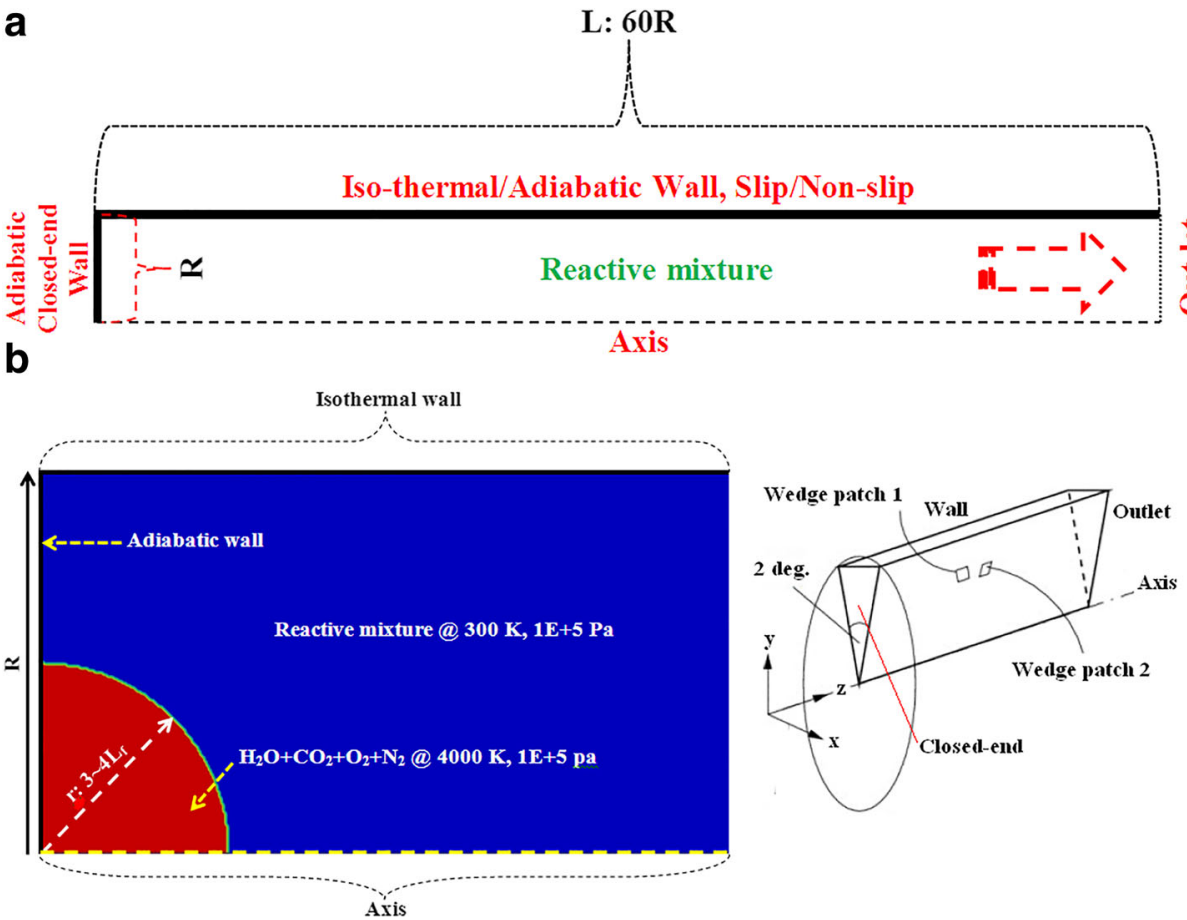

Fig. 1 a Schematic view of the applied geometry and physical domain in the current study. $\mathbf{b}$ Schematic view of the defined geometry (right) and the applied initial kernel for commencing the ignition (coordinate axes: $\mathrm{y}$ (radial direction) $-\mathrm{x}$ (axial direction))

modeling. As depicted in Fig. 1b, the initial temperature $\left(T_{0}\right)$ and pressure $\left(P_{0}\right)$ of the reactive mixture in the tube are $300 \mathrm{~K}$ and $10^{5} \mathrm{~Pa}$, respectively. Also shown is the initial hot kernel of burnt gas applied for simulating the ignition. It is believed that such an ignition

Table 1 Parameters used in the simulations

\begin{tabular}{ll}
\hline Parameter & Value \\
\hline Applied mixture & Propane-air \\
Equivalence ratio $(\Phi)$ & 0.7 \\
Initial pressure & $1 \mathrm{bar}$ \\
Initial temperature & $300 \mathrm{~K}$ \\
Preheat zone thickness $\left(L_{\mathrm{f}}\right)$ & $8.339 \times 10^{-5} \mathrm{~m}$ \\
Tube radius $(R)$ & $10-30 L_{\mathrm{f}}$ \\
Tube length $(L)$ & $60 R$ \\
Wall temperature $\left(T_{\mathrm{w}}\right)$ & $300-900 \mathrm{~K}$ \\
Kernel's initial radius & $3-4 L_{\mathrm{f}}$ \\
Kernel's initial temperature & $4000 \mathrm{~K}$ \\
Lewis number of the unburnt mixture $(\mathrm{Le})$ & $\sim 1.85$ \\
\hline
\end{tabular}


initiation is similar to reality. As seen in Fig. 1b, the kernel's (hemispherical) radius $r$ is about 3-4 times the unstretched laminar adiabatic flame speed $L_{\mathrm{f}}$.

In this study, due to the fact that only the early stages of burning are modeled $(0<t<$ $0.005 \mathrm{~s})$, it is assumed that the flow is completely laminar and simultaneously compressible due to significant variation in density of the gases before and after the flame front $(\Theta)$, which is in the range 6-8. In other words, due to the fact that evolving the flame front from the kernel shape to a finger shape in the tube pushes the unburned reactive mixture towards the outlet, therefore, for flow that is laminar when $\operatorname{Re}<2300$ and turbulent when $\operatorname{Re}>4000$, it is in transient form when the Reynolds number is in-between. In this study, it is seen that the flow field is mostly laminar in the tube under the various boundary conditions applied, for the simulation time $t<0.005 \mathrm{~s}$.

The time-dependent conservation equations of mass, axial and radial momentum, energy, and species are solved for the laminar, compressible, 2D-axisymmetric, and transient reactive, multi-species flows as follows:

- Continuity

$$
\frac{\partial \rho}{\partial t}+\frac{\partial\left(\rho u_{i}\right)}{\partial x_{i}}=0
$$

- Species conservation (see Appendix for details)

$$
\frac{\partial\left(\rho Y_{k}\right)}{\partial t}+\frac{\partial\left(\rho u_{i} Y_{k}\right)}{\partial x_{i}}+\frac{\partial\left(\rho V_{i}^{\mathrm{c}} Y_{k}\right)}{\partial x_{i}}+\frac{\partial\left(\rho W_{k, i} Y_{k}\right)}{\partial x_{i}}=\dot{\omega}_{k}+\frac{\partial\left(\rho D_{k m} \frac{\partial Y_{k}}{\partial x_{i}}\right)}{\partial x_{i}}
$$

- Momentum

$$
\left(\frac{\partial \rho u_{i}}{\partial t}+\frac{\partial \rho u_{j} u_{i}}{\partial x_{j}}\right)=-\frac{\partial p}{\partial x_{i}}+\frac{\partial}{\partial x_{j}}\left[\mu_{\operatorname{mix}}\left(\frac{\partial u_{i}}{\partial x_{j}}+\frac{\partial u_{j}}{\partial x_{i}}\right)-\frac{2}{3} \mu_{\operatorname{mix}} \frac{\partial u_{k}}{\partial x_{k}} \delta_{i j}\right]+\rho f_{i}
$$

In Eqs. 8-10, the indices $i, j$, and $k$ label the directions ( $z$ or axial), with the exception of Eq. 9, where $k$ indicates one of the five species: $\mathrm{C}_{3} \mathrm{H}_{8}, \mathrm{O}_{2}, \mathrm{~N}_{2}, \mathrm{CO}_{2}$, or $\mathrm{H}_{2} \mathrm{O}$. In Eq. 10, the viscosity of a mixture $\mu_{\operatorname{mix}}$ was calculated from Wilke's mixture rule [21]:

$$
\begin{gathered}
\mu_{\text {mix }}=\sum_{i=1}^{n} \frac{X_{i} \mu_{i}}{\sum_{j=1}^{n} X_{i} \varphi_{i j}}, \\
\varphi_{i j}=\frac{\left[1+\left(\frac{\mu_{i}}{\mu_{j}}\right)^{1 / 2}\left(\frac{M_{j}}{M_{i}}\right)^{1 / 4}\right]^{2}}{2 \sqrt{2}\left(1+\frac{M_{i}}{M_{j}}\right)^{1 / 2}} .
\end{gathered}
$$

The dynamic viscosity of each species is calculated using Sutherland's equation as [22]

$$
\mu_{i}=\frac{A_{\mathrm{S}} \sqrt{T_{i}}}{1+\frac{T_{\mathrm{S}}}{T_{i}}}
$$


where the coefficients $A_{\mathrm{S}}$ and $T_{\mathrm{S}}$ usually are $1.67212 \times 10^{-6}$ and 170.672 , respectively. Also, the equation for conservation of energy in the gas phase is, in terms of the sensible enthalpy $h_{\mathrm{s}}$ is

$$
\begin{aligned}
\frac{\partial\left(\rho h_{\mathrm{s}}\right)}{\partial t}+\frac{\partial\left(\rho u_{i} h_{\mathrm{s}}\right)}{\partial x_{i}}+\frac{\partial\left(\rho V_{i}^{\mathrm{c}} h_{\mathrm{s}}\right)}{\partial x_{i}}= & \\
\frac{\mathrm{D} p}{\mathrm{D} t}-\sum_{k=1}^{n} \dot{\omega}_{k} h_{\mathrm{s}, k}+ & \frac{\partial}{\partial x_{i}}\left(\rho \alpha_{\text {mix }} \frac{\partial h_{\mathrm{s}}}{\partial x_{i}}\right)+\frac{\partial}{\partial x_{i}}\left[\rho \alpha_{\operatorname{mix}} \sum_{k=1}^{n}\left(\frac{1}{\mathrm{Le}_{k}}-1\right) h_{\mathrm{s}, k} \frac{\partial Y_{k}}{\partial x_{i}}\right] \\
& -\frac{\partial}{\partial x_{i}}\left(\rho \sum_{k=1}^{n} h_{\mathrm{s}, k} Y_{k} W_{k, i}\right)+\dot{Q}
\end{aligned}
$$

where

$$
\alpha_{\text {mix }}=\frac{k_{\text {mix }}}{\rho_{\text {mix }} c_{p, \text { mix }}}, \quad k_{\text {mix }}=\sum_{i=1}^{n} \frac{X_{i} k_{i}}{\sum_{j=1}^{n} X_{i} \varphi_{i j}},
$$

with $\varphi_{i j}$ given by Eq. 12 [21].

In the study, it is assumed that the gas-phase species behave as ideal gases. Thus, the thermal equation of state becomes

$$
p=\rho R_{\mathrm{u}} T \sum_{k=1}^{N} \frac{Y_{k}}{M_{k}} .
$$

The mole and mass fractions are related by

$$
X_{k}=\frac{Y_{k} \bar{M}}{M_{k}}=\frac{Y_{k} / M_{k}}{\sum_{l=1}^{N}\left(Y_{l} / M_{l}\right)}
$$

with

$$
\bar{M}=\sum_{k=1}^{N} X_{k} M_{k}
$$

The temperature $T$ is obtained from the caloric equation of state,

$$
h_{\mathrm{s}}=\int_{T_{\mathrm{std}}}^{T} C_{p} \mathrm{~d} T \text {. }
$$

Here, $T_{\text {std }}$ is the standard temperature, which is defined based on the formation enthalpies, usually $T_{\text {std }}=298.15 \mathrm{~K}$. The standard-state thermodynamic properties of ideal gases being mostly dependent on temperature, the standard molar specific heat at constant pressure for species $k$ can be calculated as

$$
\frac{C_{p, k}^{\circ}}{R_{\mathrm{u}}}=\sum_{n=0}^{4} a_{n, k} T^{n},
$$

where the superscript ${ }^{\circ}$ denotes the standard state. Similarly, the standard-state values of the molar enthalpy $\left(H_{k}^{\circ}\right)$ and the standard-state molar entropy $\left(S_{k}^{\circ}\right)$ can be computed by

$$
\frac{H_{k}^{\circ}}{R_{\mathrm{u}} T}=\sum_{n=0}^{4} \frac{a_{n, k} T^{n}}{n+1}+\frac{a_{5, k}}{T}
$$

and

$$
\frac{S_{k}^{\circ}}{R_{\mathrm{u}}}=A_{0, k} \log (T)+\sum_{n=1}^{4} \frac{a_{n, k} T^{n}}{n}+a_{6, k} .
$$


Further, due to the fact that the specific heats and the enthalpy of ideal gases are mostly dependent on temperature, thus, in the above equations, the superscript ${ }^{\circ}$ of $C_{p, k}^{\circ}$ and $H_{k}^{\circ}$ can be dropped.

In the current study, the numerical computations were carried out using a finite volume scheme, using the OpenFOAM CFD toolbox [23] (http://www.openfoam.com), to solve the transient, laminar, and reactive flows. The pressure-velocity coupling was modeled using PIMPLE, which is a merged type of SIMPLE (Semi-Implicit Method for Pressure-Linked Equations) and PISO (Pressure-Implicit Split Operator) algorithms developed into a collocated mesh. In this study, for the Navier-Stokes, energy, and species conservation equations, the time derivatives were solved with an Euler scheme (first-order, bounded, implicit), the convective term (divergence) with Gauss [linear interpolation (central differencing), MUSCL (Monotonic Upstream-Centered Scheme for Conservation Laws), and improved version of MUSCL with $\psi=0$ ), the diffusive term (Laplacian) with Gauss (linear orthogonal), the gradient with Gauss (second order, Gaussian integration, second order, unbounded], and the interpolation terms with linear interpolation (central differencing). The accuracy of approximation for all diffusive and convective terms is of 2 nd order. The density matrix was solved by a diagonal solver. Also, the preconditioned conjugate gradient solver (PCG) was chosen to solve the pressure matrix. For velocity, enthalpy, kinetic energy, and dissipation field matrices, a preconditioned bi-conjugate gradient (PBiCG) solver was used. Moreover, in this study, the rhoReactingFoam solver of OpenFOAM was applied. This solver, which is developed based on the density-based thermodynamics package, can be efficiently used for modeling the time-dependent combustion processes accompanied by several chemical reactions. The fluid-flow chemistry interaction was modeled using a laminar, finite rate scheme. Therefore, in the applied libraries, the ordinary differential equations (ODEs) system was solved using the semi-implicit Bulirsch-Stoer (SIBS) chemistry solvers. This is a reliable and efficient solver which is recommended for stiff systems of ODEs. Furthermore, it should be noted that in the applied code, the effect of internal and external radiation was ignored.

In order to keep the computation time reasonable while acquiring general knowledge about the flame propagation in isothermal tubes, the fuel-lean propane-air chemical reactions in the combustion domain were calculated by a single-step irreversible global reaction model as $[7,8,17-20]$

$$
\mathrm{C}_{3} \mathrm{H}_{8}+\frac{5}{0.7}\left(\mathrm{O}_{2}+3.76 \mathrm{~N}_{2}\right)->3 \mathrm{CO}_{2}+4 \mathrm{H}_{2} \mathrm{O}+26.857 \mathrm{~N}_{2}+2.143 \mathrm{O}_{2} .
$$

As mentioned previously in the literature [7, 8, 17-20], a single-step irreversible global reaction is still valid for presenting the effects of external perturbations on the flame front behavior in such tubes. Therefore, according to the above reaction, the fuel consumption rate $\left(\dot{\omega}_{\mathrm{C}_{3} \mathrm{H}_{8}}\right)$ is given by the Arrhenius reaction rate

$$
\dot{\omega}_{\mathrm{C}_{3} \mathrm{H}_{8}}=-A \exp \left(-\frac{T_{a, \mathrm{C}_{3} \mathrm{H}_{8}}}{T}\right)\left[\mathrm{C}_{3} \mathrm{H}_{8}\right]^{0.1}\left[\mathrm{O}_{2}\right]^{1.65},
$$

where the activation temperature is $T_{\mathrm{a}}=E_{\mathrm{a}} / R_{\mathrm{u}}=15106 \mathrm{~K}$ for propane, with the activation energy $E_{\mathrm{a}}=1.256 \times 10^{5} \mathrm{~J} / \mathrm{mol}[7,8]$. This reaction model fundamentally follows the study of Westbrook and Dryer [24]. It should be noted that the pre-exponential factor $A$ should be re-adjusted to match the burning velocity of the working reactive mixture since it depends on several parameters such as computational mesh sizes, equivalence ratio, and the method of evaluating mixture properties. In our case, the value used is $2.40 \times 10^{12}\left[\frac{\mathrm{cm}^{3}}{\mathrm{~mol} \mathrm{~s}^{1.333}}\right]^{0.75}$. Also, it should be mentioned that the application of the single-step irreversible global 
reaction model could significantly reduce the stiffness of the system of equations and the computation time.

In the present study, the characteristics of the early stages of flame propagation in a fuellean $(\Phi=0.7)$ propane-air mixture from a kernel shape to a wrinkled/stretched flame front in isothermal tubes is investigated. According to [20], the unstretched laminar adiabatic flame speed $S_{\mathrm{L}}$ of such a mixture with an equivalence ratio of 0.7 is about $0.217 \mathrm{~m} / \mathrm{s}$. As illustrated in Fig. 1a, we consider a one-ended tube, with the closed end an adiabatic wall, while the outside wall is at a constant temperature (in the range 300 to $900 \mathrm{~K}$ ), with either a non-slip or slip boundary condition for velocity. The pressure at the open end of the tube is set to $10^{5} \mathrm{~Pa}$. Specifically, the boundary conditions on the physical domain are set as follows:

$$
\begin{aligned}
& x_{1}=0 \rightarrow \begin{cases}u_{i}=0 \rightarrow & \text { (non }- \text { slip condition }) \text { or } \frac{\partial u_{i}}{\partial x_{i}}=0 \text { (slip condition) } \\
\frac{\partial T}{\partial x_{i}}=0, & \frac{\partial Y_{j}}{\partial x_{i}}=0, \quad \frac{\partial P}{\partial x_{i}}=0\end{cases} \\
& x_{2}=R \rightarrow\left\{\begin{array}{l}
u_{i}=0 \text { (non }- \text { slip condition) } \text { or } \frac{\partial u_{i}}{\partial x_{i}}=0 \text { (slipcondition) } \\
T=T_{\mathrm{w}} \text { (constant) } \\
\frac{\partial Y_{j}}{\partial x_{i}}=0, \quad \frac{\partial P}{\partial x_{i}}=0
\end{array}\right. \\
& x_{1}=L \rightarrow\left\{\begin{array}{l}
\frac{\partial u_{i}}{\partial x_{i}}=0, \quad \frac{\partial Y_{j}}{\partial x_{i}}=0, \quad \frac{\partial T}{\partial x_{i}}=0 \\
P=\left\{\begin{array}{l}
\text { Wave Transmissive } \\
\text { uniform } \sim 10^{5} \mathrm{~Pa} \\
\gamma=1.4 \\
L_{\text {inf }}=10^{50} \mathrm{~m} \\
\text { field }_{\text {inf }}=10^{5} \mathrm{~Pa}
\end{array}\right.
\end{array}\right.
\end{aligned}
$$

with $i=1,2,3$ labelling the dimensions and $j=1, \ldots, n$ the chemical species. The other surfaces have been considered as empty faces. The initial condition corresponds to a hemispherical ignition kernel, using the Zeldovich-Frank-Kamenetsky solution [25] for the distribution of temperature,

$$
\frac{T}{T_{\mathrm{u}}}=1+(\Theta-1) \exp \left(-\frac{\xi-R_{0}}{L_{\mathrm{f}}}\right)
$$

where $\xi \equiv r^{2}+x^{2}$, and chemical species,

$$
Y=\frac{\Theta-\frac{T}{T_{\mathrm{u}}}}{\Theta-1} .
$$

\section{Validation}

Validation of the numerical approach used in this work can be done using several methods, including making comparisons with the available experimental data (e.g., [19]) and with the relevant theory as presented in [6] and summarized in Section 2. This is shown in Fig. 2a and $\mathrm{d}$, for the particular case of the early stages of burning of the fuel-lean propane-air mixture (with an equivalence ratio of 0.7 ) in a tube with adiabatic walls. It should be noted that in the present study, the flame tip position along the tube axis $\left(Z_{\text {tip }}\right)$ is determined from where the propane concentration on the symmetry axis starts to decline. 

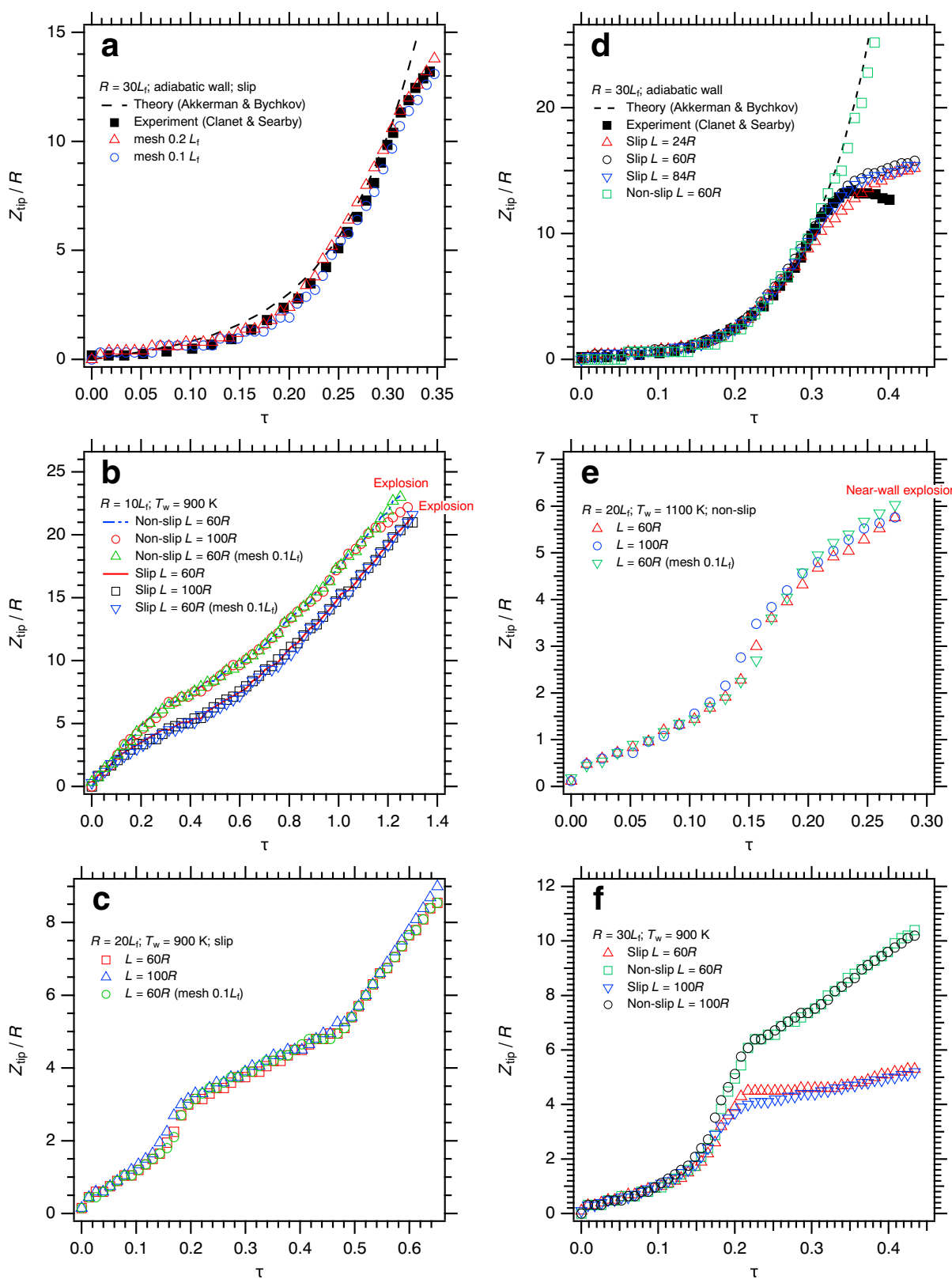

Fig. 2 Propagation of a propane-air flame front in a cylindrical tube with isothermal or adiabatic walls, for different tube length $L$ and radius $R$, slip/non-slip walls, wall temperatures $T_{\mathrm{w}}$, and mesh sizes $\left(0.2 L_{\mathrm{f}}\right.$, unless noted otherwise). Comparison is made to the experimental results of Clanet and Searby [19] and the theory of Bychkov et al. [6]

We see from Fig. 2 that the numerical simulation satisfactorily predicts the flame position and its propagation behavior in comparison to experimental and theoretical results. Also, it can be inferred that compared to the experimental results [19], the numerical scheme used 
Table 2 Number of computational cells used for the various Reynolds numbers

\begin{tabular}{ll}
\hline $\operatorname{Re}$ & Total number of computational cells \\
\hline 10 & 150000 \\
20 & 600000 \\
30 & 1350000 \\
\hline
\end{tabular}

in the present study can predict the flame propagation regime in the adiabatic tube more accurately than previous simulations [6]. According to the results of the present study of the flame acceleration region (Fig. 2a,d; $\tau<0.35$ ), the maximum deviation of the numerical results from the experimental ones is acceptably about $8 \%$. However, it increases to $20 \%$ at time $\tau=0.4$, probably due to a violation the assumption of adiabatic walls in the experimental tests [19].

\subsection{Mesh size}

In order to increase the precision of the computations, the effect of the mesh size was conducted based on the procedures described by Zarvandi et al. [26], Baigmohammadi et al. [27-29], Alipoor and Mazaheri [30], Akkerman et al. [31-33], and Bychkov et al. [6, 3436]. Therefore, as shown in Fig. 2a, two uniform structured quad meshes with cell sizes of $0.2 L_{\mathrm{f}} \times 0.2 L_{\mathrm{f}}$ (denoted $\left.0.2 L_{\mathrm{f}}\right)$ and $0.1 L_{\mathrm{f}} \times 0.1 L_{\mathrm{f}}\left(\right.$ denoted $\left.0.1 L_{\mathrm{f}}\right)$, with $L_{\mathrm{f}}=8.339 \times 10^{-5} \mathrm{~m}$, were examined and their respective non-dimensional flame tip positions were calculated. It can be clearly seen in Fig. 2 that the refinement of the mesh sizes to $0.1 L_{\mathrm{f}}$ has no appreciable effect on the final results of the simulation. In order to keep computation times within a reasonable range, subsequent calculations were performed using a cell size of $0.2 L_{\mathrm{f}}$. Table 2 presents the number of computational cells used for the various Reynolds numbers.

\subsection{Length of the tube}

As previously mentioned in the literature [16], tube length may affect flame propagation regimes in non-adiabatic tubes. In fact, there is a minimum tube length that makes the flame propagation regimes in the tube independent of the tube length. Thus, in this section, for the sake of making the results independent of the tube length, the effect of increasing the tube length on the final results, especially non-dimensional flame tip position versus nondimensional time, is investigated. It can be seen in Fig. 2 that increasing the tube length from 60 times the tube radius $R$ (denoted L60R) to 100 times (denoted L100R) has no considerable effect on the results, so that it can be inferred that our results are basically insensitive to variation in the tube length. However, increasing the tube length from $60 R$ to $100 R$ almost doubles the computation times, therefore a tube length of $60 R$ has been chosen for most computations.

\section{Results}

We have studied the effects of various parameters on the early stages of burning of a fuel-lean premixed propane-air mixture. The results are presented below in three distinct subsections for improved clarity. 


\subsection{Flame propagation stages}

As partly indicated in Fig. 3, independent of the reactive mixture and all boundary conditions, the flame shape evolution from a small-size kernel to a flat or curved flame front during the early stages of burning of a still, premixed reactive mixture in a one-ended closed tube consists, generally, of four main stages [6, 19]: (1) evolution from a kernel to a hemispherical propagating flame; (2) evolution from a spherical to a finger-shaped flame front; (3) evolution from a finger-shaped flame to a tulip-shaped one; and (4) evolution from a tulip-shaped flame to a finger-shaped one. According to these flame evolution stages, there are four representative critical non-dimensional time stages which can describe the flame propagation styles at the early stages of burning in combustors. As indicated in Fig. 3, these time stages are $\tau_{\mathrm{sph}}$, where the flame front propagates in a hemispherical shape; $\tau_{\text {wall }}$, when the flame front touches the wall; $\tau_{\text {finger-tulip }}$ (Tfinger-tulip), when the flame front shape changes from a finger shape to a tulip shape; and $\tau_{\text {tulip-finger, }}$, when the flame front shape changes from a tulip shape to a finger shape again. Thus, as seen in Fig. 3 and Table 3, and according to [30], the present simulation can capture all the critical non-dimensional times (e.g., $\tau_{\text {sph }}, \tau_{\text {wall }}$, and $\tau_{\text {finger-tulip }}$ ) of the flame evolution in the tube, compared to the theory which only captures the first two stages. As seen in Table 3, the maximum deviation between the experimental and the numerical results is about $18 \%$. This deviation may be related to the effect of other parameters, such as the tube radius, non-adiabatic condition on the real tube wall, and the propane-air overall chemistry scheme which has been applied in the present study. In fact, these parameters can strongly influence the flame thickness and its propagation speed and also the effect of heat loss on the flame front in the tube. As is known, these influence factors can significantly govern the flame propagation regime in every combustion device. It should be noted here that there is an uncertainty in the computed values of $\tau$ due to the graininess of snapshots in time from our simulation.

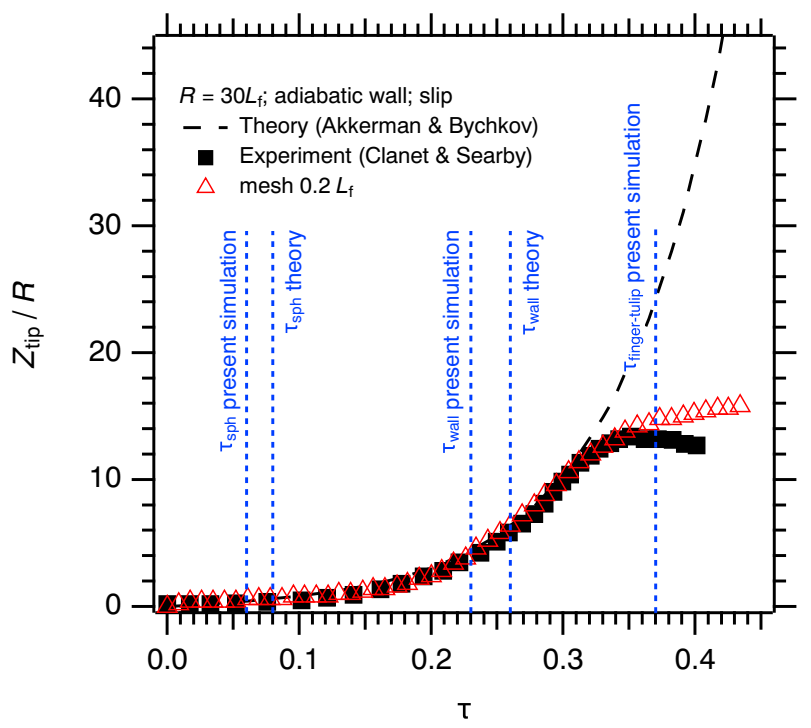

Fig. 3 Various stages of flame propagation in an adiabatic tube $\left(R=30 L_{\mathrm{f}} ; L=60 R\right.$; mesh size: $0.2 L_{\mathrm{f}}$; adibatic wall with slip condition). Comparison is made to theory [6] and experiment [19] 
Table 3 Critical non-dimensional times of the flame evolution in the tube (present study, see Fig. 3: $R=$ $30 L_{\mathrm{f}} ; L=60 R$; mesh size: $0.2 L_{\mathrm{f}}$; adiabatic wall with slip condition)

\begin{tabular}{llll}
\hline & $\tau_{\text {sph }}$ & $\tau_{\text {wall }}$ & $\tau_{\text {finger-tulip }}$ \\
\hline Experiment [19] & - & $\sim 0.28$ & $\sim 0.33$ \\
Theory [6] & $\sim 0.08$ & $\sim 0.26$ & - \\
Present study & $\sim 0.06$ & $\sim 0.23$ & $\sim 0.37$ \\
\hline
\end{tabular}

It can be seen in Fig. 3 that there is a good compatibility between the theory, the experimental data, and the numerical results of the present study for times shorter than $\tau \approx 0.3$. For $\tau>0.3$, the first signs of hydrodynamic instabilities on the flame surface appear, which makes the flame surface wrinkled. This wrinkling causes a deviation between the theoretical and the numerical and experimental results. In fact, the theory is not able to precisely predict the early stages of burning in such small-sized tubes after the point where the flame's trailing skirt touches the tube wall, due to the limitations and restrictions of the theoretical model. Thus, the folding and wrinkling of the flame front leads to a significant reduction in the flame acceleration, violating the exponential propagation of the flame front in an adiabatic tube from $\tau \approx 0.3$. A further discussion of this point is presented in the following sections.

\subsection{Effects of tube radius and wall temperature}

In this section, the effects of variations in the tube radius and the tube wall temperature on the flame propagation regime in small-size tubes are considered. In this regard, three tube radii $R$ of $10 L_{\mathrm{f}}, 20 L_{\mathrm{f}}$, and $30 L_{\mathrm{f}}$, accompanied by four uniform wall temperatures of $300 \mathrm{~K}$, $500 \mathrm{~K}, 700 \mathrm{~K}$, and $900 \mathrm{~K}$, have been chosen. The motivation for the selection of these values is based on the validity of the solutions for laminar flows and also for preventing the selfexplosion of the reactive mixture by the heated wall temperature, for the tube radius and wall temperature, respectively. The effects of these parameters is seen in Figs. 4, 5, 6, 7 and 8, with detailed results about the variation of the flame front position versus time on the tube axis presented in Fig. 4 and flame profiles at the early stages of burning plotted in Figs. 5-8, for both slip and non-slip wall conditions.

\subsubsection{Exponential and non-linear regimes of flame propagation}

It is seen in Figs. 4-8 that the early stages of burning from a small kernel in small-sized tubes with isothermal walls, independent of slip or non-slip wall conditions, are comprised of two main regimes of exponential and semi-linear flame propagation. The semi-linear part of the flame propagation in the isothermal tubes can present either a positive or a negative slope, which can consequently lead to progressive or regressive flame propagation speeds in the isothermal tubes, respectively. For instance, it can be observed in Fig. 4b that the early stages of flame propagation in a tube with the isothermal wall temperature of $300 \mathrm{~K}$ and non-slip wall condition, show first an exponential and subsequently a semi-linear trends during the time intervals $0<\tau \leq 0.3$ and $0.3<\tau \leq 0.65$, respectively. However, the semi-linear trend is disturbed or even absent when increasing the inner radius and the wall temperature of the tubes with slip walls.

It is noteworthy that, independent of either slip or non-slip condition on the closed-end of the tube, the exponential evolution of an initial ignition kernel in the isothermal-walled 
Fig. 4 Effects of the tube radius and the wall temperature on the flame propagation in a tube with isothermal walls
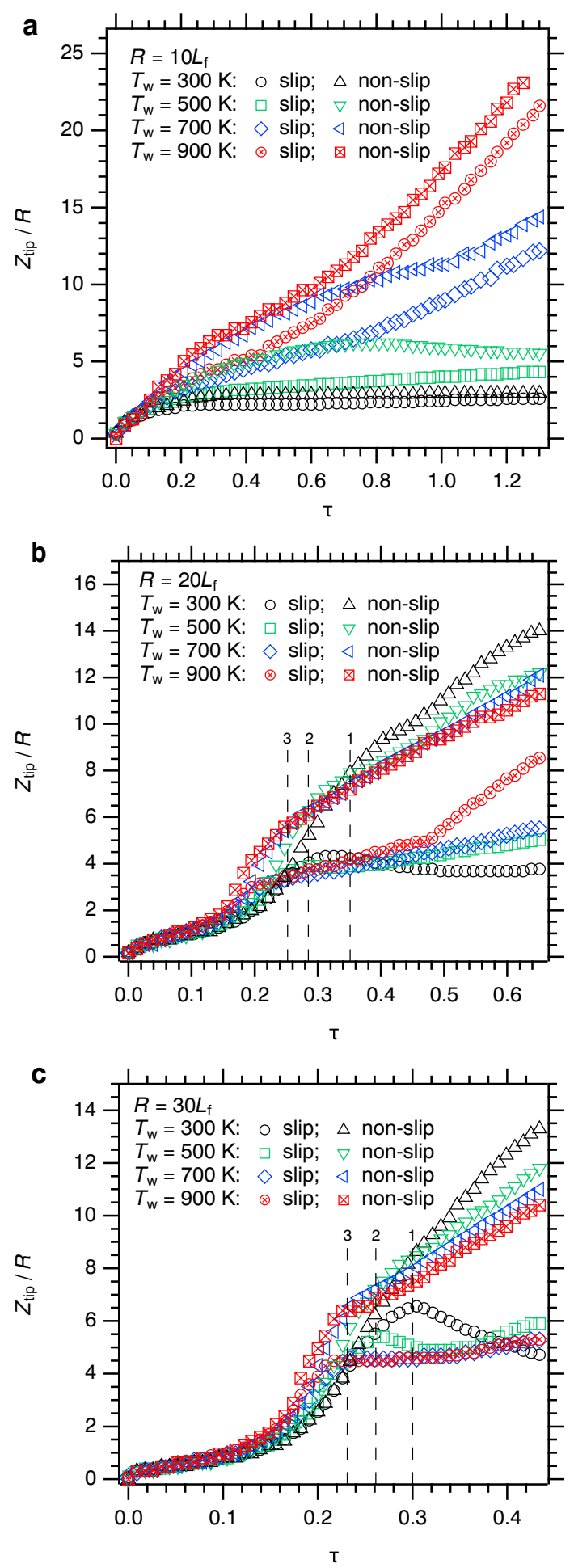


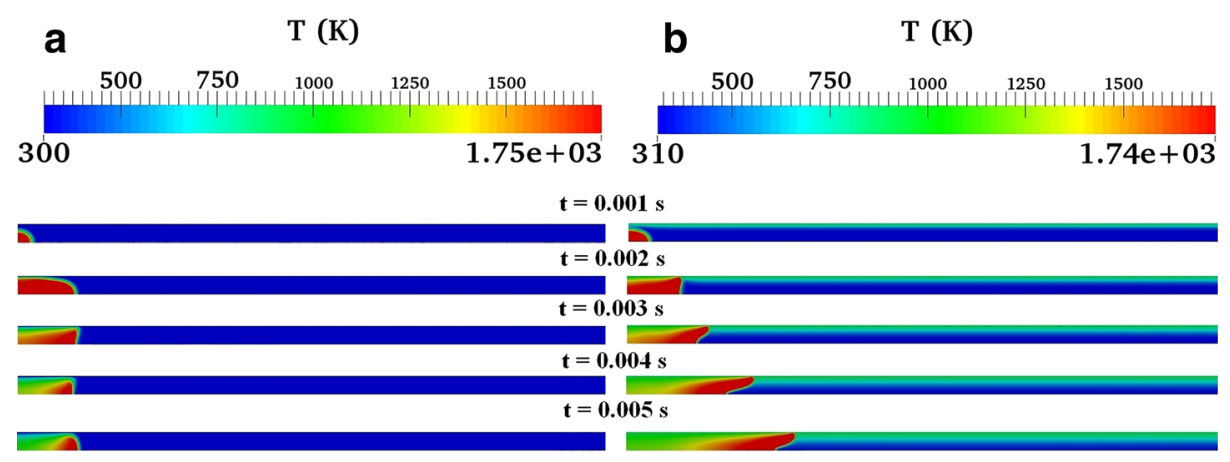

Fig. 5 Flame evolution in a tube with isothermal wall and radius of $20 \mathrm{Lf}$, with slip wall condition: $\mathbf{a} T_{\text {wall }}=$ $300 \mathrm{~K} ; \mathbf{b} T_{\text {wall }}=900 \mathrm{~K}$

tubes almost obeys the trend which was already predicted by the theory for the hemi-bubble shape propagation of the flame front at the early stages of burning before touching the wall. However, a while after the flame's trailing skirt touches the wall, the flame front gradually starts to decelerate in a semi-linear fashion due to the cooling effect of the isothermal wall on the flame temperature and also to the curvature/stretch in the tubes with either slip or non-slip walls.

\subsubsection{Effects of tube radius}

It can be seen in these results that increasing the tube radius and the wall temperature have different effects on the flame propagation in the tubes with slip or non-slip walls. It is seen in Fig. 4 that although increasing the wall temperature in a tube with $R=10 L_{\mathrm{f}}$ and either slip or non-slip walls has a monotonic increasing effect on the flame propagation speed in the tube, its effect on the flame propagation speeds in the tubes with $R=20 L_{\mathrm{f}}$ and $30 L_{\mathrm{f}}$ is completely different. It can be observed in Fig. 4 that increasing the tube radius significantly changes the flame propagation style in the early stages of the propagation in both of the isothermal tubes (with slip or non-slip walls) due to its influence on the critical time stages $\left(\tau_{\mathrm{w}}\right)$, the flame-wall interaction (heat generation versus the wall's cooling effect), and the occurrence of hydrodynamic instabilities on the flame front. In this regard, it is seen that although increasing the tube radius leads to an oscillating behavior of the flame tip position

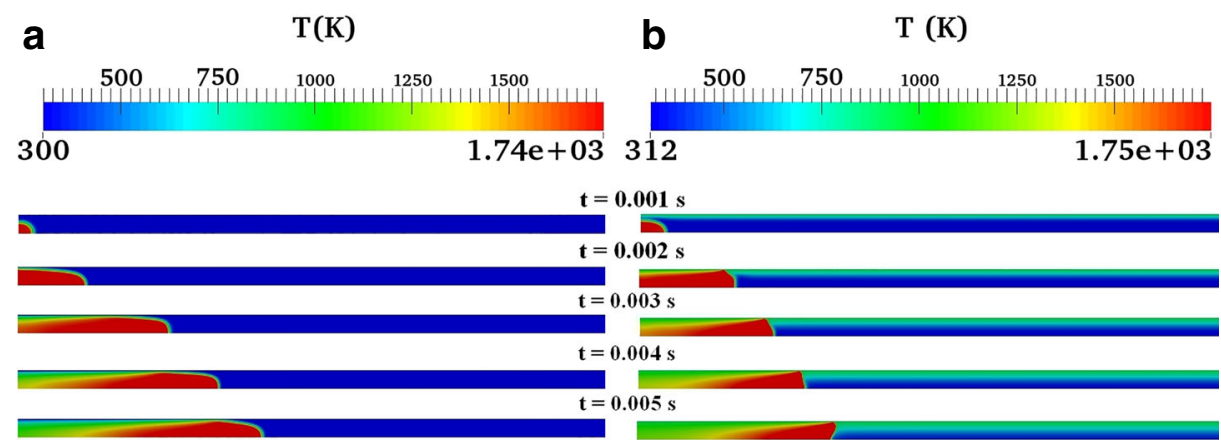

Fig. 6 Flame evolution in a tube with isothermal wall and radius of $20 \mathrm{Lf}$, with non-slip wall condition: a $T_{\text {wall }}=300 \mathrm{~K} ; \mathbf{b} T_{\text {wall }}=900 \mathrm{~K}$ 


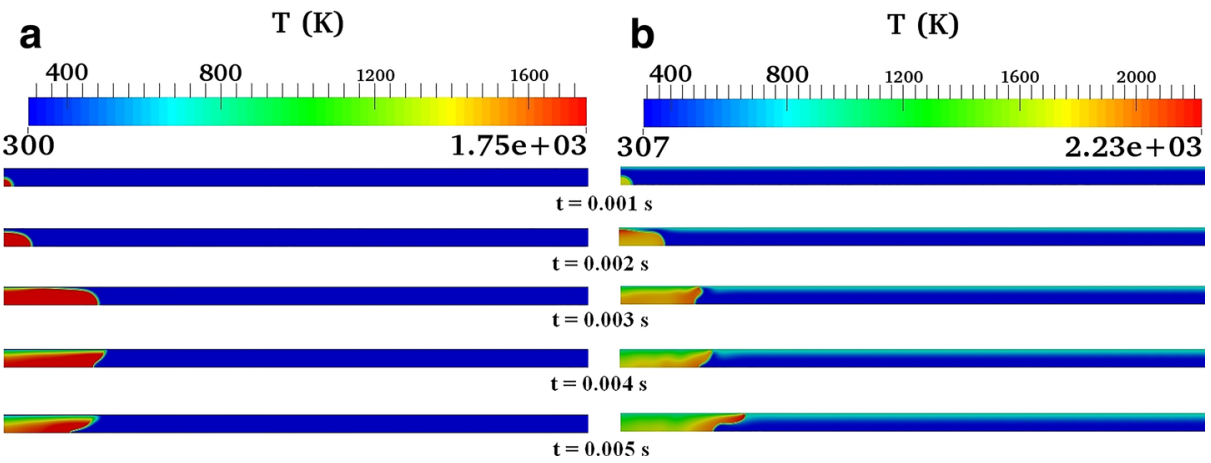

Fig. 7 Flame evolution in a tube with isothermal wall and radius of $30 L \mathrm{f}$, with slip wall condition: $\mathbf{a} T_{\text {wall }}=$ $300 \mathrm{~K} ; \mathbf{b} T_{\text {wall }}=900 \mathrm{~K}$

in the isothermal tubes with slip walls, with non-slip walls the flame tip position and propagation speed only increases with the tube radius. As seen in Figs. 5-8, increasing the tube radius makes the flame front more prone to be wrinkled by the occurrence of hydrodynamic instabilities on the flame surface which is very sensitive to the ratio between the flame thickness and the tube radius. As mentioned previously by Senchenko et al. [37], hydrodynamic instabilities may threaten the stability of a planar flame front in a gaseous fuel when the cut-off wavelength is larger than $20 L_{\mathrm{f}}$.

Also, it is depicted in Fig. 4 that increasing the tube radius can intensify the effect of the slip or non-slip condition on the flame propagation at the early stages of burning. This means that the flame evolution in the isothermal tubes with slip walls is very sensitive to perturbations which can change the positive flame stretch to a negative one. In fact, as seen in Fig. 9b, increasing radius of a tube with slip wall condition, when the flame thickness is constant, can make the flame front more prone to be bended from "position 1" to "position 2 " by any sufficient pressure gradient between the flame up- and down-stream due to an increasing ratio between the tube diameter and the flame thickness, $2 R / \delta_{\mathrm{t}}$. This matter can be intensified by the sliding of the flame front on the walls which in fact increases the flame susceptibility to such a deformation due to its reducing effect on the positive stretch of the propagating flame front in the isothermal tubes. Such an analysis can be verified by the flame profiles depicted Figs. 5-8. Unlike the slip wall condition, as can be seen in Fig. 9c,

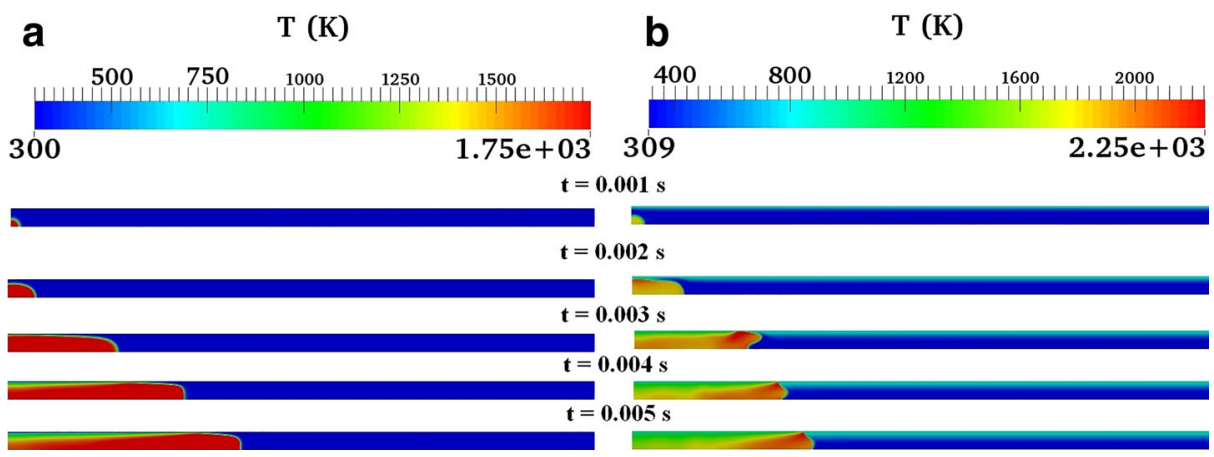

Fig. 8 Flame evolution in a tube with isothermal wall and radius of $30 \mathrm{Lf}$, with non-slip wall condition: a $T_{\text {wall }}=300 \mathrm{~K}$; b $T_{\text {wall }}=900 \mathrm{~K}$ 


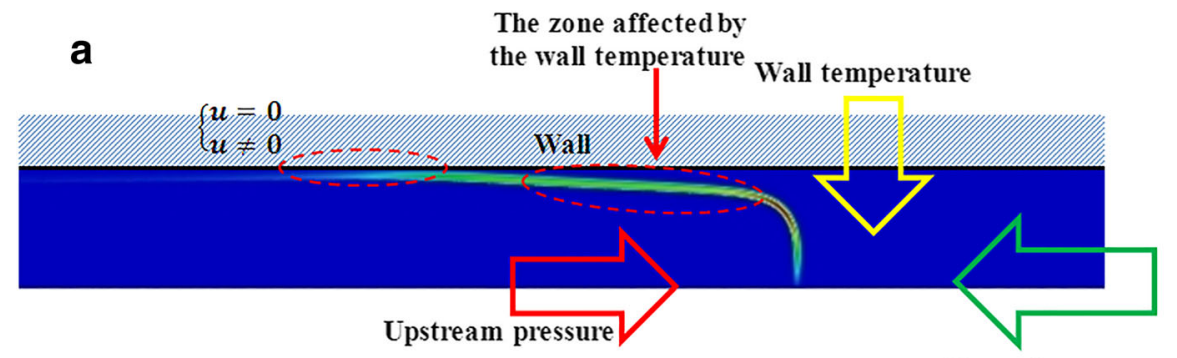

Downstream pressure

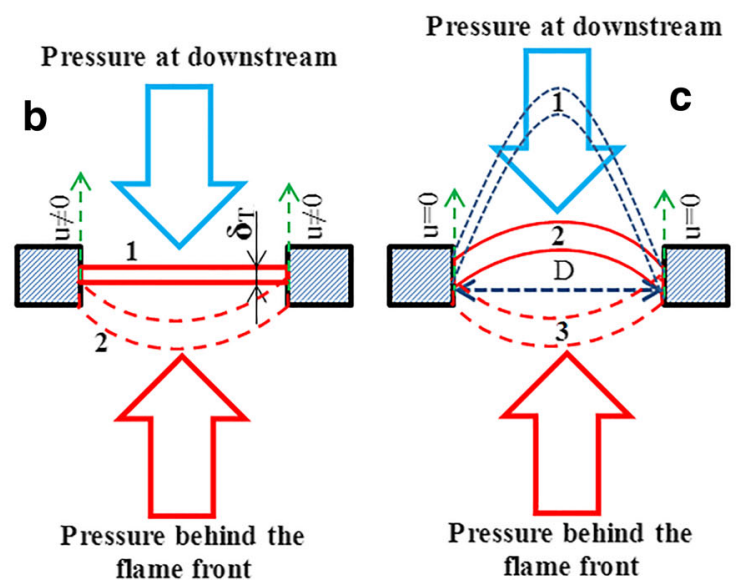

Fig. 9 Schematic representation of the effects of various parameters on flame stretch in a tube (see text for details)

the non-slip wall condition can significantly increase the flame strength against changing the flame shape from "position 1" to "position 3" due to the increasing effect of the non-slip wall on the positive stretch of the flame front. This effect can be intensified by increasing the tube radius, which can lead to an increased effect on the flame positive curvature/stretch and the ratio between the heat generation and the wall cooling rates in the tubes with the non-slip walls, as support by the results in Figs. 5-8.

Furthermore, we observe in Fig. 4 that increasing the radius of the tubes with slip conditions and elevated temperature isothermal walls $\left(T_{\text {wall }} \geq 500 \mathrm{~K}\right)$ disturbs the flame propagation after touching the wall of tubes with a radius $\geq 20 L_{\mathrm{f}}$. This is due to the fact that the simultaneous increase of the tube radius and its wall temperature can significantly intensify the occurrence of hydrodynamic instabilities in the tubes with slip walls due to a reduction in the flame thickness and the preheating of the still, reactive mixture. As seen in Figs. 5-8, under such a condition, elevating the slip wall temperature can significantly increase the flame negative stretch due to boosting the flame propagation speed in the vicinity of the tube wall. Indeed, as depicted in Fig. 9b, increasing the tube radius accompanied by increasing the wall temperature makes the flame front more prone to be easily deformed from "position 1" to "position 2" due to an increase in the flame speed near the wall and also an increase of the ratio between the flame thickness and the tube radius. Therefore, the superposition of these conditions can cause more complex time-varying flame shapes in the tubes which can lead to such an irregular oscillatory flame propagation in the tubes with elevated-temperature slip walls. 
Moreover, it is seen in Fig. 4 that although increasing the radius of a tube with non-slip wall intensifies the effect of wall temperature on the flame propagation speed in the tube, its effect attenuates the influence of the wall temperature on the flame propagation speed in the tubes with the slip walls. The main reason of such a phenomenon can be found in Figs. 5-8. It can be inferred that by increasing the tube radius, the flame curvature and consequently its propagation speed in the tubes with non-slip walls are almost governed by the effect of wall temperature on the flame curvature in the tube, while the flame curvature and its propagation speed in the tubes with slip walls are almost controlled by the tube radius. In fact, as mentioned above, according to the ratio between the flame thickness and the tube radius, increasing the tube radius increases the possibility of hydrodynamic instabilities on the flame surface in the tubes with either slip or non-slip walls. As is known, the occurrence of hydrodynamic instabilities can increase or decrease flame propagation speed in the tubes by increasing or decreasing the flame curvature/surface in the tubes. Under such a condition, it can be seen that the effect of wall temperature on the flame surface and curvature is more pronounced by increasing the tube radius due its significant effect on the flame curvature and its propagation speed near the wall. Unlike tubes with non-slip walls, one can observe in Figs. 5-8 that the effect of the wall temperature on the flame surface and curvature in the tubes with slip walls is decreased by increasing the tube radius due to more sensitivity of the flame front to tube radius compared to the wall temperature. In fact, by increasing the radius of the tubes with slip walls, resulting in the appearance of a hydrodynamic instabilities on the flame surface in such tubes, the effect of the wall temperature on the flame shape and curvature is mostly changed from a primary role in the tubes with non-slip walls to a supportive one in the tubes with slip walls, which can intensify the change in the flame shape, especially near the wall.

\subsubsection{Effects of wall temperature}

From Fig. 4b and c, we see also that although increasing the non-slip wall temperature increases the flame propagation speed in the exponential part, it has a decreasing effect on the linear part of the flame acceleration in the early stages of burning in the isothermal wall tubes with non-slip wall condition. In this regard, it can be seen in Figs. 5-8 that, on one hand, increasing the wall temperature can increase the flame propagation speed in the exponential part $\left(<\tau_{\mathrm{w}}\right)$ due to the preheating effect of the wall temperature on the reactive mixture which can consequently lead to a more stretched flame front with much faster propagation speed in the tubes. On the other hand, increasing the wall temperature can reduce the flame propagation speed in the linear part due to its decreasing effect on the flame curvature and stretch in the tube. As seen in Figs. 5-8, an increase of the flame propagation speed near the non-slip tube wall reduces the flame's positive stretch (reducing the flame's surface), causing a significant reduction in the flame speed in such tubes. As seen at $\tau=0.003 \mathrm{~s}$ in Fig. 8b, this difference between the flame propagation speed on the tube axis and near the tube wall can fold the flame front, slowing the propagation of the flame front in the tubes with non-slip walls.

\subsubsection{Effect of wall velocity boundary condition}

The effect of the wall velocity boundary condition (slip or non-slip) on the flame tip position on the tube axis is shown in Fig. 2. It can be seen in Fig. 2d that imposing the non-slip condition on the wall can exponentially increase the flame acceleration in an adiabatic tube, while the slip wall condition can significantly suppress flame acceleration, especially after 
that the flame skirt touches the walls. As schematically illustrated in Fig. 9a-c, this can be due to the fact that imposing the non-slip wall condition can increasingly strengthen the flame front against the flame folding and negative flame stretch in the tubes. In other words, the non-slip walls can significantly increase the positive stretch of the flame front towards the unburned mixture in the tubes so that, as depicted in Fig. 2d, such a positive stretched flame front can almost follow the theory trend for predicting the flame propagation in the adiabatic tube due to exponential effect of the positive stretched flame front on the flame propagation in the tube. It should be noted here that the flame stretch is positive when its convexity is towards the unburned mixture and inversely its stretch is negative when its concavity is towards the unburned mixture. Thus, it is seen in Fig. 2 that imposing the nonslip condition on the tube wall, which is in close contact with the still, reactive mixture in the tube, can increase the flame acceleration at the early stages of burning by at least more than a factor of two compared to the slip condition. (For a theoretical analysis of the phenomenon, see, e.g., [38-40].)

Here, we should note that although the presence of wrinkling/stretch on the flame surface, as shown in Fig. 10, can increase the flame surface, it also changes the local burning rate. In this regard, one can see in Fig. 10 that the heat release in the vicinity of the wall is higher than at the center. In fact, increasing the flame stretch and the flame surface has two distinct effects on the flame front propagation regimes in confined chambers. The effect of flame stretch on the flame propagation style in a confined chamber can be affected by parameters such as slip/non-slip wall, heat/non-heated wall, positive or negative stretch conditions, Lewis number, diffusion time scales, steady or non-steady propagation, etc. [41-43] It has been already shown by Bell et al. [41] and Tseng et al. [43] how changing in the flame stretch could affect the wrinkling pattern on the flame surface and the flame's local propagation speed in accordance with the Lewis number and the Markstein length.

In contrast to the non-slip case, isothermal tubes with the slip wall condition has an increasing-decreasing effect on the flame acceleration at the early stages of burning in the tubes due to complex interactions between the decreasing flame curvature/stretch and the increasing effect of the mixture preheating on the flame speed in the tubes. In fact, by considering Figs. 5-8, it can be inferred that increasing the slip wall temperature can disturb and (sometimes) reduce the flame propagation speed in the tubes due to intensifying distortions and folding of the flame surface. In addition, it is seen in Fig. $4 \mathrm{~b}$ and c that increasing the non-slip wall temperature moves the starting point of the linear part of the flame propagation to shorter times, due to the increased propagation speed in the earlier exponential part because of the elevated wall temperature.

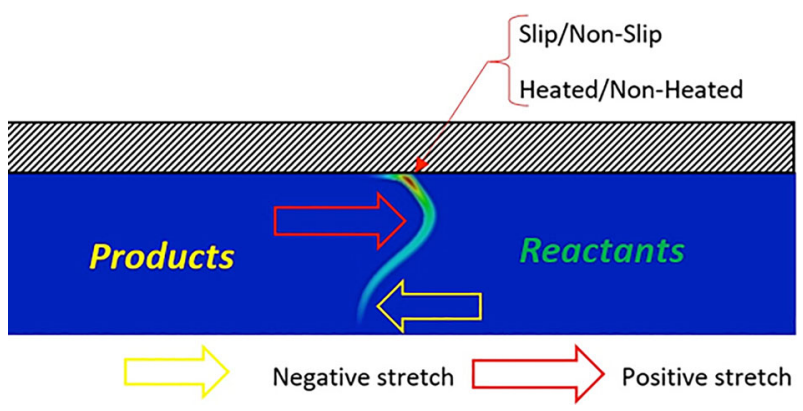

Fig. 10 Schematic of a wrinkled flame surface showing the heat release contour 
Furthermore, by comparing Figs. 5b and 6, it can be inferred that slip and non-slip wall conditions can strongly affect the flame propagation speed in the isothermal tubes due to a significant effect on the flame shape at the early stages of burning. Figure 5 illustrates that increasing the slip wall temperature can impressively influence the flame shape near the wall due to the significant increase of the burning rate in the vicinity of the tube wall and also increasing the flame susceptibility to perturbations caused by pressure oscillations in the tube. In combination, they strongly change the flame shape in the time interval $t=0.001 \mathrm{~s}$ to 0.004 s. For non-slip walls, Fig. 6, the effect of elevated wall temperature is reduced, leading to less distortion of the flame surface. As already shown in Fig. 9, the non-slip wall condition can significantly increase the flame strength against perturbations caused by pressure oscillations in the tubes by imposing an increasing positive stretch on the flame surface. In this regard, it can be observed in Figs. 11 and 12 that there is an accumulated pressure in the flame downstream when the flame front starts to have a negative stretch or tulip shape. Indeed, the presence of a notable difference in pressure down- and up-stream makes the flame front prone to bending, depending on the ratio between the flame thickness and the tube radius, slip or non-slip wall conditions, positive or negative flame stretch, and the wall temperature. As seen in Fig. 11, the presence of accumulated pressure in the flame downstream during the time interval $t=0.0015 \mathrm{~s}$ to $0.0025 \mathrm{~s}$, significantly influences the flame shape in a tube with a slip wall at a temperature of $900 \mathrm{~K}$, since all conditions are present for such a flame behavior.

Unlike the slip wall condition, it can be seen in Figs. 6 and 12 that the non-slip wall condition can postpone the transition of the flame front shape from the positive stretch to the negative one in the last time stages $(t>0.0045 \mathrm{~s})$. In this regard, Fig. 12 shows that using non-slip wall condition completely disturbs the pressure field in the tube, so that there is not a significant gradient in the pressure field when the flame front is changed from the positive stretch to the negative one. However, it is seen that, compared to the slip wall case, the pressure accumulation in the tube is increased by passing the time before that the flame shape is changed to the negatively stretched flame front. Also, it can be understood from
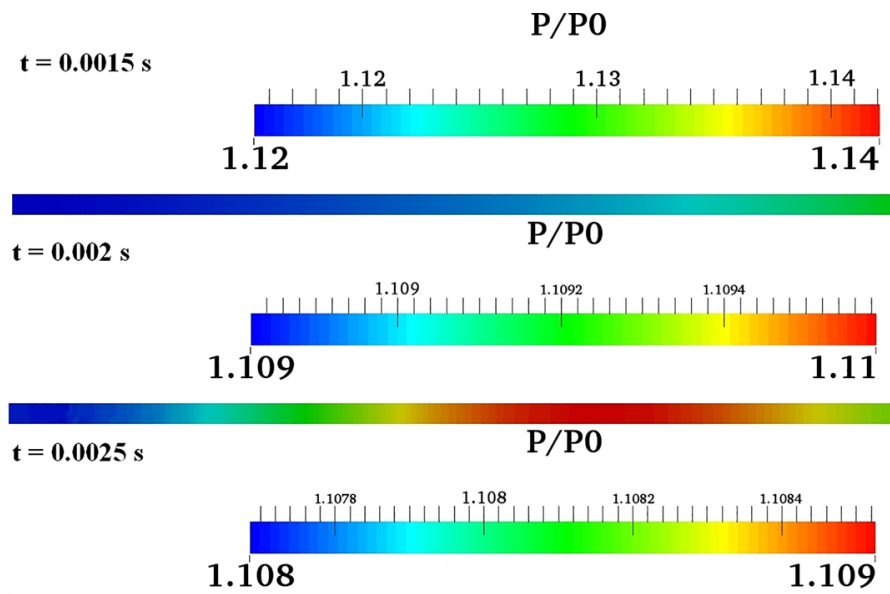

Fig. 11 Pressure in a tube with radius of $20 L_{\mathrm{f}}$, slip wall condition, wall temperature of $900 \mathrm{~K}$ 


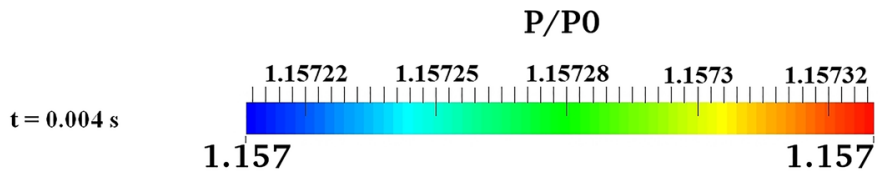

\begin{tabular}{|c|}
\hline$t=0.0045 \mathrm{~s}$ \\
\hline
\end{tabular}

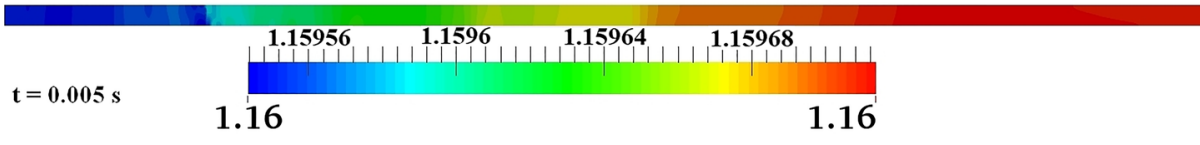

Fig. 12 Pressure in a tube with radius of $20 L_{\mathrm{f}}$, non-slip wall condition, wall temperature of $900 \mathrm{~K}$

Fig. 12 that as compared to the tubes with slip walls, changing the flame shape from the positively stretched flame front to the negatively stretched one happens in higher pressures accompanied by a smaller gradient in the pressure field in the tubes with non-slip walls.

\subsubsection{Interplay between wall temperature and slip/non-slip conditions}

It is seen in Fig. 6 that increasing the temperature of a non-slip wall (from $300 \mathrm{~K}$ to $900 \mathrm{~K}$ ) significantly reduces the flame surface and simultaneously increases the flame susceptibility to various instabilities which can lead to extensive wrinkles on the flame surface. As mentioned above, this effect of wall temperature on the flame shape can be related to various parameters such as the increasing effect of wall temperature on the mixture burning rate near the wall and also reducing the ratio between the flame thickness and the tube radius which can significantly intensify the occurrence possibility of various instabilities (especially of hydrodynamic type) in the tubes. Therefore, as discussed before, independent of slip or non-slip wall conditions, this effect can significantly disturb the flame propagation at the early stages of burning in the isothermal tube with elevated wall temperature. Similar behavior can be seen in Fig. 8b. However, by comparing Figs. 6 and 8b, one can maintain that the transition from positively stretched flame front to negatively stretched can happen at te earlier time stages of the flame evolution $(0.002 \mathrm{~s}<t<0.003 \mathrm{~s})$ due to an increase of $2 R / \delta_{\mathrm{t}}$ and of the pressure gradient between the flame up- and down-stream in the tube. The latter can be inferred by a simple comparison of Figs. 12 and 13.

In addition, Fig. 7 shows that increasing the temperature of a slip wall (from $300 \mathrm{~K}$ to $900 \mathrm{~K}$ ) has an oscillating effect on the flame surface in the tubes. As seen in Fig. 7a, the flame front curvature in a tube with a cold slip wall $\left(T_{\text {wall }}=300 \mathrm{~K}\right)$ is changed from positively stretched to negatively stretched between times $t=0.003 \mathrm{~s}$ and $t=0.004 \mathrm{~s}$, so that it leads to a significant reduction in the flame surface. However, the flame surface is gradually increases from $t=0.004 \mathrm{~s}$ to $t=0.005 \mathrm{~s}$. By increasing the wall temperature to $900 \mathrm{~K}$, one can see in Fig. 7b that the transition of the stretched flame front from the positively stretched to negatively stretched occurs earlier, between $t=0.002 \mathrm{~s}$ and $t=0.003 \mathrm{~s}$, due to the elevated wall temperature increasing the mixture burning rate near the wall and 

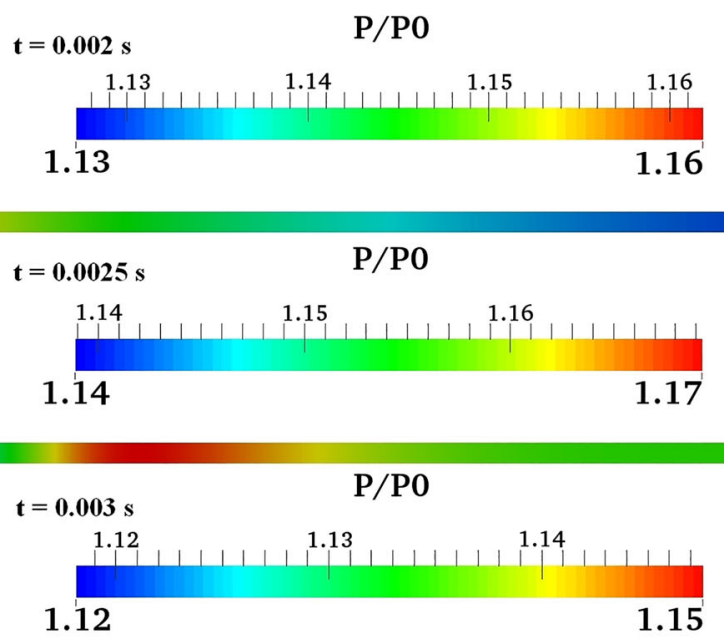

Fig. 13 Pressure in a tube with radius of $30 L_{\mathrm{f}}$, non-slip wall condition, wall temperature of $900 \mathrm{~K}$

decreasing the flame thickness, which increases $2 R / \delta_{\mathrm{t}}$. Under such conditions, the flame surface is decreased to its minimum value at $t=0.003 \mathrm{~s}(\tau \approx 0.26)$. Afterwards $(t>0.003 \mathrm{~s})$, the surface of the negatively stretched flame front starts to grow again. Such a behavior leads to an oscillation of the flame position and its propagation style in the tubes with isothermal slip walls. In this regard, further information can be obtained by considering the pressure field in the tubes, Figs. 14 and 15. We see again that the flame front deformation from positively to negatively stretched occurs when there is enough accumulated pressure in the flame downstream in the tube. This accumulated pressure leads to a positive pressure gradient in the tube between the closed end and the open one which, in certain conditions, can deform the flame shape in tubes with slip walls. One can also see in these figures that increasing the slip wall temperature in a tube of radius $30 L_{\mathrm{f}}$ increases the accumulated

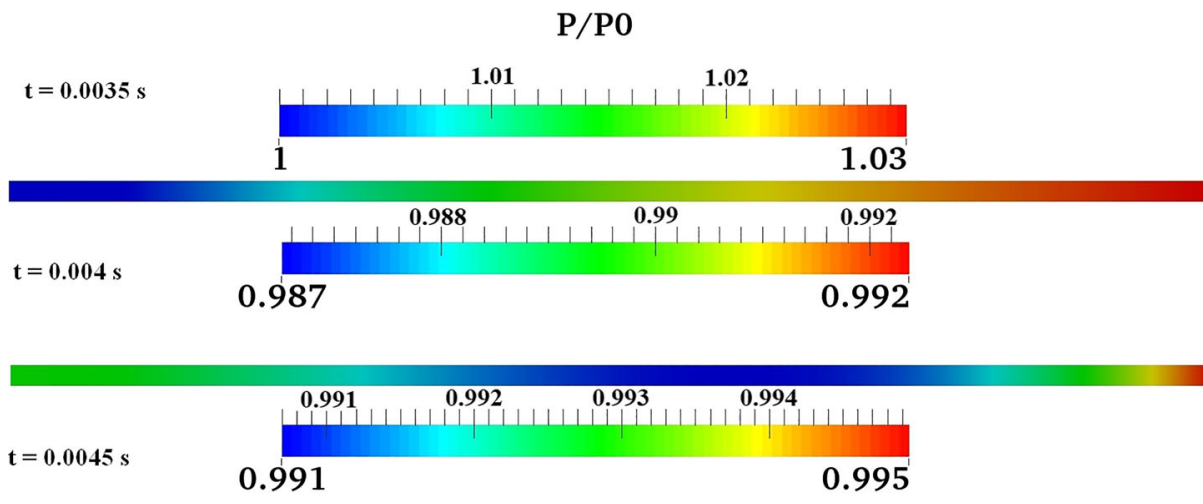

Fig. 14 Pressure in a tube with radius of $30 L_{\mathrm{f}}$, slip wall condition, wall temperature of $300 \mathrm{~K}$ 

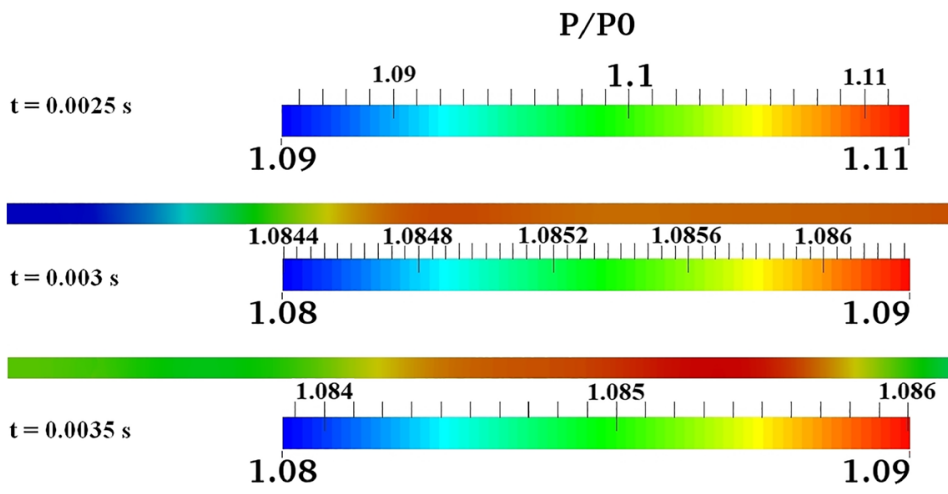

Fig. 15 Pressure in a tube with radius of $30 L_{\mathrm{f}}$, slip wall condition, wall temperature of $900 \mathrm{~K}$

pressure level in the tube when the flame front deformation happens. For instance, the critical accumulated pressure, in a tube where the flame front deformation has occurred, is changed from $\sim 1-1.03$ in Fig. $14(t=0.0035 \mathrm{~s})$ to $\sim 1.09-1.11$ in Fig. $15(t=0.0025 \mathrm{~s})$. Such a behavior is, to a lesser extent, also observed in the other cases presented here.

Furthermore, it is shown in Figs. 7 and 8 how imposing the non-slip wall condition can influence the flame shape and its propagation style in the tubes with isothermal wall. However, the effect of non-slip walls is more prominent in tubes with the cold walls $\left(T_{\text {wall }}=\right.$ $300 \mathrm{~K}$ ). In this regard, we seen that imposing the non-slip wall condition completely suppresses the flame's negative stretch in the tubes with cold walls, while its effect on the transition time from the positively stretched flame front to the negatively stretched one in the tubes with the elevated wall temperature $\left(T_{\text {wall }}=900 \mathrm{~K}\right)$ is not obvious (Figs. $7 \mathrm{~b}$ and $8 \mathrm{~b})$. However, one can observe in Fig. $8 \mathrm{~b}$ that, between $t=0.003 \mathrm{~s}$ and $t=0.005 \mathrm{~s}$, the flame curvature gradually returns to a positive stretch, probably because of the supportive effect of the non-slip condition and wall temperature on the flame speed in the tube. In fact, as seen in Fig. 13, such a flame behavior occurs due to the positive support of the pressure field in the flame upstream, pushing the flame front downstream.

\subsubsection{Non-slip condition and pressure}

Finally, by comparing Figs. 13 and 15, we can infer that imposing the non-slip wall condition increases the level of the critical accumulated pressure (from 1.11 to 1.17) and also the pressure gradient between the closed end and the open one (from $\sim 0.02$ to $\sim 0.03$ ) required to deform the flame front curvature in the tube. This is in accord with the analysis presented in Fig. 9. It can be understood from Figs. 11 to 15 that increasing the wall temperature and imposing a non-slip wall condition can increase the critical pressure required for flame front deformation due to their effect on the pressure field upstream. In fact, as reported previously $[4,44]$, the cold wall is principally responsible for decreasing to pressure upstream of the flame, due to the condensing water vapor of the combustion products. This phenomenon intensifies the positive pressure gradient between the closed end and the open one in the tube, which can lead to the flame deformation from a positively stretched flame front to a negatively stretched. Therefore, it can be concluded that imposing the nonslip condition and simultaneously increasing the wall temperature can reduce this effect in 
the tubes by reducing the cooling effect of the wall temperature on the combustion products and also increasing the rate of combustion process in the tube caused by increasing the flame positive stretch in the tubes.

\section{Conclusion}

In the present study, the problem of fuel-lean premixed propane-air flame propagation in the early stages of burning in semi-long narrow tubes from the closed to open end for the case of cold and hot isothermal tube walls has been numerically investigated. In this regard, simultaneous effects of the elevated wall temperatures $(\gg 300 \mathrm{~K})$, tube diameter, and slip or non-slip conditions on flame configurations in small scale, one-end-closed tubes/channels have been examined in detail.

The results showed that imposing the non-slip condition on the wall could exponentially increase the flame acceleration in an adiabatic tube, while imposing the slip wall condition could significantly suppress flame acceleration, especially after that the flame skirt touched the walls. Moreover, it was demonstrated that the early stages of burning from a small size kernel in the small size tubes with isothermal walls, independent of either slip or nonslip wall conditions, were comprised of two main regimes of exponential and semi-linear propagation. In this regard, it was shown that although increasing the tube radius had an oscillatory effect on the flame tip position (distance from the closed end) in the isothermal tubes with slip walls, its effect on the flame tip position and its propagation speed in isothermal tubes with non-slip walls was increasing. Also, the results showed that increasing the tube radius could intensify the effect of slip or non-slip conditions on the flame propagation at the early stages of burning. It was seen that increasing the radius of the tubes with slip and elevated temperature isothermal walls disturbed the flame propagation style after touching the wall of tubes with a radius larger than $20 L_{\mathrm{f}}$. We also showed that although increasing the radius of a tube with a non-slip wall intensified the effect of the wall temperature on the flame propagation speed in the tube, it attenuated the effect of the wall temperature on the flame propagation speed in the tubes with the slip walls. Unlike tubes with non-slip walls, we could observe that the effect of wall temperature on the flame surface and curvature in the tubes with slip walls was decreased by increasing the tube radius. Also, it was shown that although increasing the non-slip wall temperature had an increasing effect on the flame propagation speed in the exponential part, it had a decreasing effect on the linear part of the flame acceleration at the early stages of burning in the isothermal wall tubes with non-slip wall condition. Moreover, unlike the isothermal tubes with non-slip walls, increasing the wall temperature in the isothermal wall tubes with slip wall conditions had an oscillatory effect on the flame acceleration at the early stages of burning in the tubes. Furthermore, it could be inferred that slip and non-slip wall conditions could strongly affect flame propagation speed in the isothermal tubes due to significant effects on the flame shape at the early stages of burning. Unlike the flame propagation style in the tubes with slip walls, it was shown that the flame shape in the tubes with elevated temperature non-slip walls was less sensitive to the effect of wall temperature on the flame shape.

Moreover, we demonstrated that increasing the temperature of a non-slip wall significantly reduced the flame surface and simultaneously increased the flame susceptibility to various instabilities which could lead to extensive wrinkles on the flame surface. As shown, the flame front deformation from a positively stretched flame front to a negatively stretched one occurred when there was enough accumulated pressure in the flame downstream in the tube. Furthermore, it could be inferred that imposing the non-slip wall condition could 
obviously increase the level of the critical accumulated pressure and the pressure gradient between the closed-end and the opened one in the tubes which were required for deforming the flame front curvature in the tube.

As future work, we suggest that using a detailed chemical mechanism instead of an overall chemistry (as used in this study) can be considered as an approach for a high-fidelity modeling of similar physics in small-size tubes/channels. In fact, using the detailed chemical mechanisms could more precisely predict the flame-wall interactions in small-size tubes/channels where the flame front is exposed to high heat loss rates. Also, adding a thickened wall instead of an infinitely thin isothermal wall which could interact with outer ambient through convective and radiative heat transfer and circulate the heat generated in the combustion zone to the unburned reactive mixture could be applied as an effective parameter for a better modeling of the flame acceleration in the small-scale tubes/channels.

Acknowledgements The authors dedicate this article to the memory of Pr Vitaly Bychkov.

Funding Information Open access funding provided by Umea University. The present work was supported financially by the Swedish Research Council (VR), the Kempe Foundation and the Faculty of Natural Sciences, Umeå University. The computations were performed on resources provided by the Swedish National Infrastructure for Computing (SNIC) at HPC2N, Umeå University.

\section{Compliance with Ethical Standards}

Conflict of interests No potential conflict of interest was reported by the authors.

Open Access This article is distributed under the terms of the Creative Commons Attribution 4.0 International License (http://creativecommons.org/licenses/by/4.0/), which permits unrestricted use, distribution, and reproduction in any medium, provided you give appropriate credit to the original author(s) and the source, provide a link to the Creative Commons license, and indicate if changes were made.

\section{Appendix : Species Conservation}

For species conservation, we start from

$$
\frac{\partial\left(\rho Y_{k}\right)}{\partial t}+\frac{\partial}{\partial x_{i}}\left[\rho Y_{k}\left(u_{i}+V_{k, i}+W_{k, i}\right)\right]=\dot{\omega}_{k},
$$

where $V_{k, i}$ is the diffusion velocity corresponding to the $k$-th species. Here, it was assumed that the mass diffusion follows the Fickian rule, i.e.,

$$
V_{k, i}=-\frac{D_{k m}}{Y_{k}} \frac{\partial Y_{k}}{\partial x_{i}}
$$

In this study, the Soret and Dufour effects have been assumed to be negligible, thus $\left.W_{(} k, i\right)$, which is referring to the Soret effect, is zero. From Eq. 27, the equation of continuity is rewritten as

$$
\frac{\partial \rho}{\partial t}+\frac{\partial\left(\rho u_{i}\right)}{\partial x_{i}}+\left(\rho \sum_{k=1}^{N} V_{k, i} Y_{k}\right)=0
$$

with

$$
\sum_{k=1}^{N} V_{k, i} Y_{k}=0
$$


implying the mass diffusion fluxes

$$
V_{k, i}=V_{k, i}^{*}+V_{i}^{\mathrm{c}}
$$

with

$$
V_{k, i}^{*}=-\frac{D_{k m}}{Y_{k}} \frac{\partial Y_{k}}{\partial x_{i}}, \quad V_{i}^{\mathrm{c}}=\sum_{k=1}^{N} D_{k m} \frac{\partial Y_{k}}{\partial x_{i}} .
$$

Thus, we can rewrite Eq. 27 as

$$
\frac{\partial\left(\rho Y_{k}\right)}{\partial t}+\frac{\partial}{\partial x_{i}}\left[\rho Y_{k}\left(u_{i}+-\frac{D_{k m}}{Y_{k}} \frac{\partial Y_{k}}{\partial x_{i}}+V_{i}^{\mathrm{c}}\right)\right]=\dot{\omega}_{k}
$$

to finally get the species conservation as Eq. 9, with

$$
D_{k m}=\frac{1-X_{k}}{\sum_{j \neq k}^{n} \frac{X_{j}}{D_{k j}}}
$$

where $D_{k j}$, the binary diffusion coefficient of the $k$-th species in the $j$-th species, can be calculated using the Chapman-Enskog relation [45] as follows:

$$
\begin{gathered}
D_{k j}=0.0188 \frac{\left[T^{3}\left(\frac{1}{M_{w}, k}+\frac{1}{M_{w}, j}\right)\right]^{0.5}}{p \sigma_{k j} \Omega_{D}}, \\
\sigma_{k j}=\frac{\sigma_{k}+\sigma_{j}}{2}
\end{gathered}
$$

where $\sigma$ is the collision diameter in ångström,

$$
\begin{gathered}
\Omega_{D}=\frac{1.06036}{T_{N}^{0.15610}}+\frac{0.193}{\exp \left(0.47635 T_{N}\right)}+\frac{1.03587}{\exp \left(1.52996 T_{N}\right)}+\frac{1.76474}{\exp \left(3.89411 T_{N}\right)}, \\
T_{N}=\frac{T}{E_{k j}}, \quad E_{k j}=\frac{\varepsilon_{k j}}{k_{\mathrm{B}}}
\end{gathered}
$$

with $k_{\mathrm{B}}=1.38064852 \times 10^{-23} \mathrm{~J} / \mathrm{K}$ the Boltzmann constant and $\varepsilon$ the Lennard-Jones energy, $\varepsilon_{k j}=\sqrt{\varepsilon_{k} \varepsilon_{j}}$.

\section{References}

1. Dorofeev, S.B.: Flame acceleration and explosion safety applications. Proc. Combust. Inst. 33, 2161$2175(2011)$

2. Law, C.K.: Combustion Physics. Cambridge University Press, Cambridge (2006)

3. Roy, G.D., Frolov, S.M., Borisov, A.A., Netzer, D.W.: Pulse detonation propulsion: challenges, current status, and future perspective. Prog. Energy Combust. Sci. 30, 545-672 (2004)

4. Ciccarelli, G., Dorofeev, S.: Flame acceleration and transition to detonation in ducts. Prog. Energy Combust. Sci. 34, 499-550 (2008)

5. Hsu, Y.-C., Chao, Y.-C., Chung, K.-M.: Flame propagation in $\mathrm{CH}_{4} / \mathrm{H}_{2} / \mathrm{O}_{2}$ blended mixtures in smooth tubes of millimeter-scale. Combust. Sci. Technol. 188, 1239-1248 (2016)

6. Bychkov, V., Akkerman, V., Fru, G., Petchenko, A., Eriksson, L.-E.: Flame acceleration in the early stages of burning in tubes. Combust. Flame 150, 263-276 (2007)

7. Gutkowski, A.: Numerical analysis of flame behavior near the quenching conditions during passage from wider to narrower tube diameters. Combust. Sci. Technol. 184, 1616-1634 (2012)

8. Gutkowski, A.: Numerical analysis of effect of ignition methods on flame behavior during passing through a sudden contraction near the quenching conditions. Appl. Therm. Eng. 54, 202-211 (2013)

9. Demirgok, B., Ugarte, O., Valiev, D., Akkerman, V.: Effect of thermal expansion on flame propagation in channels with nonslip walls. Proc. Combust. Inst. 35, 929-936 (2015) 
10. Liberman, M., Kuznetsov, M., Ivanov, A., Matsukov, I.: Formation of the preheated zone ahead of a propagating flame and the mechanism underlying the deflagration-to-detonation transition. Phys. Lett. A 373, 501-510 (2009)

11. Kurdyumov, V.N., Matalon, M.: Self-accelerating flames in long narrow open channels. Proc. Combust. Inst. 35, 921-928 (2015)

12. Kurdyumov, V.N., Matalon, M.: Effects of gas compressibility on the dynamics of premixed flames in long narrow adiabatic channels. Combust. Theory Model. 20, 1046-1067 (2016)

13. Kurdyumov, V.N., Matalon, M.: Critical conditions for flame acceleration in long adiabatic channels closed at their ignition end. Proc. Combust. Inst. 36, 1549-1557 (2017)

14. Song, Z.B., Wei, L.J., Wu, Z.Z.: Effects of heat losses on flame shape and quenching of premixed flames in narrow channels. Combust. Sci. Technol. 180, 264-278 (2007)

15. Dobrego, K., Kozlov, I., Vasiliev, V.: Flame dynamics in thin semi-closed tubes at different heat loss conditions. Int. J. Heat Mass Transfer 49, 198-206 (2006)

16. Kerampran, S., Desbordes, D., Veyssière, B.: Study of the mechanisms of flame acceleration in a tube of constant cross section. Combust. Sci. Technol. 158, 71-91 (2000)

17. Kim, N.I., Maruta, K.: A numerical study on propagation of premixed flames in small tubes. Combust. Flame 146, 283-301 (2006)

18. Kim, N.I.: Effect of an inlet temperature disturbance on the propagation of methane-air premixed flames in small tubes. Combust. Flame 156, 1332-1338 (2009)

19. Clanet, C., Searby, G.: On the tulip flame phenomenon. Combust. Flame 105, 225-238 (1996)

20. Davis, S., Quinard, J., Searby, G.: Markstein numbers in counterflow, methane- and propane- air flames: a computational study. Combust. Flame 130, 123-136 (2002)

21. Poling, B.E., Prausnitz, J.M., O'Connell, J.P. The Properties of Gases and Liquids, 5th edn. McGrawHill, New York (2001)

22. Sutherland, W.: LII. The viscosity of gases and molecular force. Phil. Mag. 36, 507-531 (1893)

23. Weller, H.G., Tabor, G., Jasak, H., Fureby, C.: A tensorial approach to computational continuum mechanics using object-oriented techniques. Comput. Phys. 12, 620-631 (1998)

24. Westbrook, C.K., Dryer, F.L.: Simplified reaction mechanisms for the oxidation of hydrocarbon fuels in flames. Combust. Sci. Technol. 27, 31-43 (1981)

25. Bychkov, V., Akkerman, V., Valiev, D., Law, C.K.: Role of compressibility in moderating flame acceleration in tubes. Phys. Rev. E 81, 026309 (2010)

26. Zarvandi, J., Tabejamaat, S., Baigmohammadi, M.: Numerical study of the effects of heat transfer methods on $\mathrm{CH}_{4} /\left(\mathrm{CH}_{4}+\mathrm{H}_{2}\right)$-AIR pre-mixed flames in a micro-stepped tube. Energy 44, 396-409 (2012)

27. Baigmohammadi, M., Sadeghi, S.S., Tabejamaat, S., Zarvandi, J.: Numerical study of the effects of wire insertion on $\mathrm{CH}_{4}$ (methane)/AIR pre-mixed flame in a micro combustor. Energy 54, 271-284 (2013)

28. Baigmohammadi, M., Tabejamaat, S., Kashir, B.: A numerical study on the effects of hydrogen addition levels, wall thermal conductivity and inlet velocity on methane/air pre-mixed flame in a micro reactor. Energy Equip. Syst. 2, 103-119 (2014)

29. Baigmohammadi, M., Tabejamaat, S., Zarvandi, J.: Numerical study of the behavior of methanehydrogen/air pre-mixed flame in a micro reactor equipped with catalytic segmented bluff body. Energy 85, 117-144 (2015)

30. Alipoor, A., Mazaheri, K.: Studying the repetitive extinction-ignition dynamics for lean premixed hydrogen-air combustion in a heated microchannel. Energy 73, 367-379 (2014)

31. Akkerman, V., Bychkov, V.: Turbulent flame and the Darrieus-Landau instability in a three-dimensional flow. Combust. Theory Model. 7, 767-794 (2003)

32. Akkerman, V., Bychkov, V.: Velocity of weakly turbulent flames of finite thickness. Combust. Theory Model. 9, 323-351 (2005)

33. Akkerman, V., Bychkov, V., Petchenko, A., Eriksson, L.-E.: Flame oscillations in tubes with nonslip at the walls. Combust. Flame 145, 675-687 (2006)

34. Bychkov, V.V., Golberg, S.M., Liberman, M.A., Eriksson, L.E.: Propagation of curved stationary flames in tubes. Phys. Rev. E 54, 3713-3724 (1996)

35. Bychkov, V., Akkerman, V.: Explosion triggering by an accelerating flame. Phys. Rev. E 73, 066305 (2006)

36. Bychkov, V., Petchenko, A., Akkerman, V.: On the theory of turbulent flame velocity. Combust. Sci. Technol. 179, 137-151 (2007)

37. Senchenko, S., Bychkov, V., Liberman, M.: Stability limits of curved stationary flames in cylindrical tubes. Combust. Sci. Technol. 166, 109-130 (2001)

38. Bychkov, V., Petchenko, A., Akkerman, V., Eriksson, L.-E.: Theory and modeling of accelerating flames in tubes. Phys. Rev. E 72, 046307 (2005) 
39. Akkerman, V., Bychkov, V., Petchenko, A., Eriksson, L.-E.: Accelerating flames in cylindrical tubes with nonslip at the walls. Combust. Flame 145, 206-219 (2006)

40. Demirgok, B., Sezer, H., Akkerman, V.: Flame acceleration due to wall friction: accuracy and intrinsic limitations of the formulations. Mod. Phys. Lett. B 29, 1550205 (2015)

41. Bell, J.B., Cheng, R.K., Day, M.S., Shepherd, I.G.: Numerical simulation of Lewis number effects on lean premixed turbulent flames. Proc. Combust. Inst. 31, 1309-1317 (2007)

42. Matalon, M.: Flame dynamics. Proc. Combust. Inst. 32, 57-82 (2009)

43. Tseng, L.-K., Ismail, M.A., Faeth, G.M.: Laminar burning velocities and Markstein numbers of hydrocarbonair flames. Combust. Flame 95, 410-426 (1993)

44. Dion, C.M., Demirgok, B., Akkerman, V., Valiev, D., Bychkov, V.: Acceleration and extinction of flames in channels with cold walls. 25th ICDERS (Leeds, UK) (2015)

45. Turns, S.R. An Introduction to Combustion: Concepts and Applications, 2nd edn. McGraw-Hill, New York (2000)

Publisher's Note Springer Nature remains neutral with regard to jurisdictional claims in published maps and institutional affiliations. 